\title{
Gas Phase Kinetics and Mechanistic Insights for the Reactions of Cl Atoms with Isopropyl Formate and Isobutyl Formate
}

\author{
Avinash Kumar and B. Rajakumar* \\ Department of Chemistry, Indian Institute of Technology Madras, Chennai- 600036, India. \\ *Address for correspondence: rajakumar@iitm.ac.in \\ http://chem.iitm.ac.in/faculty/rajakumar/
}

http://www.profrajakumar.com

\begin{tabular}{|c|l|c|}
\hline Sl. No. & \multicolumn{1}{|c|}{ Caption } & Page No. \\
\hline 1 & $\begin{array}{l}\text { Table S1: Vibrational frequencies of reactants, transition states, and products } \\
\text { for the reaction of isopropyl formate with Cl atoms at MP2/6-31+G (d, p) level } \\
\text { of theory. }\end{array}$ & S3-S4 \\
\hline 2 & $\begin{array}{l}\text { Table S2: Optimized geometries in Cartesian Coordinates of reactants, } \\
\text { transition states and products for the reaction of isopropyl formate with Cl } \\
\text { atoms at MP2/6-31+G (d,p) level of theory. }\end{array}$ & S5-S14 \\
\hline 3 & $\begin{array}{l}\text { Table S3: Vibrational frequencies of reactants, transition states, and products } \\
\text { for the reaction of isobutyl formate with Cl atoms at MP2/6-31+G (d, p) level } \\
\text { of theory. }\end{array}$ & S15-S18 \\
\hline 4 & $\begin{array}{l}\text { Table S4: Optimized geometries in Cartesian Coordinates of reactants, } \\
\text { transition states and products for the reaction of isobutyl formate with Cl atoms } \\
\text { at MP2/6-31+G }(\mathrm{d}, \mathrm{p}) \text { level of theory. }\end{array}$ & S19-S36 \\
\hline 5 & $\begin{array}{l}\text { Table S5: Branching ratios for the reactions of isopropyl formate with Cl } \\
\text { atoms (R1) in the temperature range of 200 - 400 K. }\end{array}$ & S36 \\
\hline 6 & $\begin{array}{l}\text { Table S6: Branching ratios for the reactions of isobutyl formate with Cl atoms } \\
\text { (R2) in the temperature range of 200 - 400 K. }\end{array}$ & S37 \\
\hline 7 & $\begin{array}{l}\text { Table S7: CVT and CVT/SCT rate coefficients for the reaction of isopropyl } \\
\text { formate with Cl atom in the temperature range of 200 - 400K at CCSD(T)/cc- } \\
\text { pVDZ/MP2/6-31+G(d, p) level of theory. }\end{array}$ & S37-S38 \\
\hline
\end{tabular}




\begin{tabular}{|c|l|c|}
\hline 8 & $\begin{array}{l}\text { Table S8: CVT and CVT/SCT rate coefficients for the reaction of isobutyl } \\
\text { formate with Cl atom in the temperature range of 200-400K at CCSD(T)/cc- } \\
\text { pVDZ//MP2/6-31+G(d, p) level of theory. }\end{array}$ & S38-S39 \\
\hline 9 & $\begin{array}{l}\text { Figure S1: IRC plots for the transition states (TS1, TS2, TS3, TS4) obtained } \\
\text { for the reaction of isopropyl formate with Cl atoms. }\end{array}$ & S39 \\
\hline 10 & $\begin{array}{l}\text { Figure S2: IRC plots for the transition states (TS1, TS2, TS3, TS4) obtained } \\
\text { for the reaction of isobutyl formate with Cl atoms. }\end{array}$ & S40 \\
\hline 11 & $\begin{array}{l}\text { Figure S3: Optimized geometries of the reactants, pre-reactive complexes, } \\
\text { transition states and products for the reaction of Cl atoms with isopropyl } \\
\text { formate obtained at MP2/6-31+G(d,p) level of theory. Black color represents } \\
\text { carbon atoms, blue color represents hydrogen atoms, green color represents Cl } \\
\text { atoms and red color represents oxygen atoms. }\end{array}$ & S41 \\
\hline 12 & $\begin{array}{l}\text { Figure S4: Optimized geometries of the reactants, pre-reactive complexes, } \\
\text { transition states and products for the reaction of Cl atoms with isobutyl formate } \\
\text { obtained at MP2/6-31+G(d,p) level of theory. Black color represents carbon } \\
\text { atoms, blue color represents hydrogen atoms, green color represents Cl atoms } \\
\text { and red color represents oxygen atoms. }\end{array}$ & S42-S43 \\
\hline 13 & $\begin{array}{l}\text { Figure S5: IR and Mass spectrum of each product obtained for the reaction of } \\
\text { isopropyl formate with Cl atom. }\end{array}$ & S44-S45 \\
\hline 14 & $\begin{array}{l}\text { Figure S6: IR and Mass spectrum along with chromatogram of each product } \\
\text { obtained for the reaction of isobutyl formate with Cl atom. }\end{array}$ & S46-S48 \\
\hline
\end{tabular}


Table S1: Vibrational frequencies of reactants, reactive complex, transition states, and products for the reaction of isopropyl formate with $\mathrm{Cl}$ atoms at $\mathrm{MP} 2 / 6-31+\mathrm{G}(\mathrm{d}, \mathrm{p})$ level of theory.

\begin{tabular}{|c|c|c|c|c|c|c|c|c|}
\hline IPF & RC1 & TS1 & TS2 & TS3 & TS4 & TS5 & TS6 & TS7 \\
\hline 73.1 & 33.5 & -959.5 & -1177.5 & -1167.8 & -1186.1 & -1176.3 & -1157.7 & -1183.7 \\
\hline 215.0 & 50.7 & 75.8 & 39.0 & 42.6 & 48.3 & 39.0 & 33.0 & 41.2 \\
\hline 227.5 & 77.3 & 84.0 & 69.3 & 64.7 & 56.8 & 69.3 & 87.3 & 77.1 \\
\hline 254.9 & 86.3 & 125.7 & 99.4 & 84.4 & 104.6 & 99.4 & 102.4 & 95.7 \\
\hline 284.7 & 217.5 & 193.3 & 215.3 & 217.3 & 212.0 & 215.3 & 216.0 & 197.8 \\
\hline 357.2 & 226.4 & 229.6 & 243.7 & 236.4 & 236.9 & 243.7 & 241.3 & 243.9 \\
\hline 432.2 & 256.6 & 233.1 & 264.5 & 273.2 & 269.8 & 264.5 & 275.9 & 270.9 \\
\hline 492.7 & 287.2 & 249.8 & 311.0 & 313.4 & 355.1 & 311.0 & 356.5 & 343.4 \\
\hline 761.8 & 356.7 & 301.7 & 416.6 & 408.6 & 443.7 & 416.5 & 413.9 & 403.5 \\
\hline 834.2 & 434.1 & 372.2 & 454.6 & 429.4 & 449.8 & 454.6 & 445.7 & 430.9 \\
\hline 951.2 & 493.6 & 409.5 & 480.6 & 484.7 & 493.6 & 480.7 & 502.1 & 470.6 \\
\hline 960.7 & 759.6 & 585.9 & 567.7 & 559.6 & 529.5 & 567.8 & 538.3 & 565.3 \\
\hline 979.4 & 832.7 & 782.5 & 770.9 & 765.0 & 768.9 & 770.9 & 768.7 & 764.7 \\
\hline 1042.6 & 949.3 & 833.9 & 833.0 & 824.1 & 833.0 & 833.0 & 835.8 & 831.1 \\
\hline 1158.7 & 962.5 & 944.7 & 891.0 & 890.6 & 901.9 & 891.1 & 894.3 & 881.4 \\
\hline 1206.4 & 979.6 & 971.0 & 949.9 & 935.8 & 944.3 & 949.9 & 946.8 & 933.3 \\
\hline 1227.9 & 1043.4 & 989.7 & 974.8 & 955.8 & 979.9 & 974.8 & 971.5 & 954.6 \\
\hline 1240.8 & 1155.7 & 1031.7 & 1004.6 & 980.9 & 1001.3 & 1004.3 & 988.5 & 991.7 \\
\hline 1393.2 & 1206.9 & 1068.7 & 1038.7 & 1037.8 & 1041.8 & 1038.7 & 1041.5 & 1039.3 \\
\hline 1408.1 & 1237.9 & 1085.2 & 1097.3 & 1092.8 & 1060.9 & 1097.1 & 1053.4 & 1095.4 \\
\hline 1423.7 & 1244.5 & 1167.3 & 1167.3 & 1188.3 & 1159.7 & 1167.3 & 1161.7 & 1190.0 \\
\hline 1449.9 & 1393.9 & 1205.0 & 1210.9 & 1201.1 & 1210.9 & 1210.9 & 1214.2 & 1202.7 \\
\hline 1462.1 & 1406.8 & 1262.9 & 1218.0 & 1215.4 & 1218.4 & 1218.0 & 1228.7 & 1216.6 \\
\hline 1526.0 & 1424.7 & 1306.3 & 1225.9 & 1238.0 & 1260.6 & 1225.8 & 1255.3 & 1240.6 \\
\hline 1530.4 & 1451.0 & 1425.6 & 1394.9 & 1386.7 & 1363.8 & 1394.9 & 1370.3 & 1382.9 \\
\hline 1540.8 & 1462.5 & 1434.7 & 1401.3 & 1415.1 & 1383.3 & 1401.3 & 1374.1 & 1416.9 \\
\hline 1553.3 & 1525.0 & 1453.9 & 1420.4 & 1419.8 & 1419.2 & 1420.3 & 1419.1 & 1418.1 \\
\hline 1766.1 & 1529.2 & 1507.4 & 1439.6 & 1457.1 & 1449.9 & 1439.5 & 1458.5 & 1463.7 \\
\hline 3120.9 & 1539.7 & 1519.6 & 1495.5 & 1504.8 & 1494.3 & 1495.5 & 1499.1 & 1498.0 \\
\hline 3123.9 & 1551.4 & 1526.3 & 1531.8 & 1537.1 & 1535.2 & 1531.8 & 1533.7 & 1536.5 \\
\hline 3161.9 & 1757.7 & 1544.5 & 1539.2 & 1543.1 & 1543.5 & 1539.2 & 1543.0 & 1541.0 \\
\hline 3167.1 & 3121.5 & 1789.6 & 1772.8 & 1770.0 & 1768.9 & 1772.8 & 1767.6 & 1768.9 \\
\hline 3215.6 & 3124.2 & 3109.6 & 3124.2 & 3127.1 & 3097.7 & 3124.2 & 3099.0 & 3129.9 \\
\hline 3222.9 & 3167.2 & 3115.2 & 3168.6 & 3170.5 & 3129.9 & 3168.6 & 3131.1 & 3171.2 \\
\hline 3230.4 & 3180.4 & 3182.3 & 3180.3 & 3174.4 & 3177.9 & 3180.3 & 3179.4 & 3175.0 \\
\hline \multirow[t]{4}{*}{3235.9} & 3216.9 & 3211.7 & 3194.3 & 3196.1 & 3196.9 & 3194.3 & 3200.7 & 3196.3 \\
\hline & 3224.2 & 3215.2 & 3226.7 & 3227.2 & 3228.3 & 3226.7 & 3230.3 & 3231.9 \\
\hline & 3230.8 & 3246.5 & 3235.6 & 3239.9 & 3247.8 & 3235.6 & 3244.8 & 3238.9 \\
\hline & 3235.4 & 3265.7 & 3305.5 & 3307.6 & 3307.2 & 3305.6 & 3311.0 & 3310.8 \\
\hline
\end{tabular}




\begin{tabular}{|c|c|c|c|c|c|c|c|c|c|}
\hline TS8 & PC1 & P1 & $\mathbf{P 2}$ & P3 & P4 & P5 & P6 & P7 & P8 \\
\hline-1057 & 39.1 & 69 & 72.7 & 67.2 & 75.8 & 79.1 & 79.3 & 79 & 65.9 \\
\hline 50.7 & 54.6 & 164.2 & 86.9 & 93.7 & 145.7 & 158.9 & 159 & 158.7 & 202.4 \\
\hline 71.7 & 79.6 & 177.3 & 218.6 & 216.6 & 217.6 & 211.8 & 211.8 & 211.8 & 227 \\
\hline 75.3 & 118.2 & 210.3 & 252.6 & 247.6 & 248.7 & 249.2 & 249.2 & 249.3 & 268.1 \\
\hline 209.2 & 173.0 & 247 & 272.8 & 274.6 & 275 & 278.8 & 278.9 & 278.8 & 310.1 \\
\hline 225.8 & 193.5 & 366.2 & 331.6 & 341.7 & 364.7 & 353 & 353 & 353 & 356.4 \\
\hline 271.5 & 206.6 & 382.4 & 429.3 & 425.7 & 404 & 406.2 & 406.2 & 406.3 & 424.6 \\
\hline 338.6 & 254.8 & 450.3 & 453.3 & 482 & 454.6 & 468.5 & 468.5 & 468.6 & 487.4 \\
\hline 353.6 & 362.8 & 754.4 & 533.2 & 589.1 & 537.6 & 604.5 & 604.4 & 604.5 & 743.5 \\
\hline 382.7 & 385.2 & 810.8 & 767 & 753.3 & 766.6 & 765 & 765 & 765 & 815.7 \\
\hline 435.2 & 417.8 & 968.5 & 828.8 & 842.9 & 844 & 825.9 & 825.9 & 825.9 & 943 \\
\hline 491.8 & 443.7 & 978.7 & 940 & 934 & 936.9 & 937.7 & 937.7 & 937.7 & 962.9 \\
\hline 773.8 & 469.5 & 1005.1 & 990.4 & 965.6 & 985.6 & 959.4 & 959.3 & 959.3 & 976.7 \\
\hline 830.3 & 748.2 & 1034.8 & 1041.5 & 1040.3 & 1041.9 & 1041.5 & 1041.5 & 1041.5 & 1141.4 \\
\hline 942.2 & 815.1 & 1127 & 1091.4 & 1073.7 & 1087.7 & 1075.6 & 1075.6 & 1075.7 & 1185.6 \\
\hline 964.3 & 969.8 & 1195.9 & 1161.6 & 1196.9 & 1174.4 & 1195.4 & 1195.2 & 1195.4 & 1203.5 \\
\hline 975.6 & 978.8 & 1257 & 1204.9 & 1218 & 1207.2 & 1222.5 & 1222.2 & 1222.4 & 1240.7 \\
\hline 977 & 1003.2 & 1334 & 1229.5 & 1230.1 & 1224 & 1232 & 1231.9 & 1232 & 1382.6 \\
\hline 1141.7 & 1043.1 & 1430.2 & 1375.9 & 1374.3 & 1373.4 & 1369.3 & 1369.3 & 1369.3 & 1407.1 \\
\hline 1205.1 & 1123.8 & 1448.6 & 1392.6 & 1413.2 & 1390.3 & 1410.4 & 1410.3 & 1410.4 & 1453.4 \\
\hline 1232.2 & 1226.6 & 1457.6 & 1423 & 1420.8 & 1422.8 & 1419.4 & 1419.3 & 1419.4 & 1463 \\
\hline 1241.6 & 1246.6 & 1511 & 1436.7 & 1454.4 & 1444.5 & 1459.8 & 1459.6 & 1459.8 & 1524.8 \\
\hline 1247.9 & 1339.3 & 1521 & 1506 & 1518.7 & 1506.8 & 1514 & 1514 & 1514.1 & 1529.2 \\
\hline 1388 & 1427.6 & 1529.1 & 1531 & 1538.9 & 1535.3 & 1533.7 & 1533.7 & 1533.7 & 1539.8 \\
\hline 1407 & 1453.1 & 1539.6 & 1542.2 & 1543.2 & 1544.8 & 1542.1 & 1542.1 & 1542.1 & 1552 \\
\hline 1454.1 & 1458.1 & 1774.9 & 1766.7 & 1762.9 & 1765.9 & 1764.6 & 1764.9 & 1764.7 & 1821.7 \\
\hline 1464.6 & 1510.5 & 3075.8 & 3124 & 3124.6 & 3101.7 & 3126.5 & 3126.5 & 3126.5 & 3122.5 \\
\hline 1524.4 & 1519.8 & 3078.1 & 3148.8 & 3159.3 & 3126.3 & 3159.4 & 3159.4 & 3159.4 & 3125.6 \\
\hline 1528.4 & 1527.2 & 3169.3 & 3166.2 & 3165.8 & 3164.2 & 3169.9 & 3170.1 & 3170 & 3161.3 \\
\hline 1539.8 & 1533.6 & 3182.4 & 3225.2 & 3221.7 & 3223.7 & 3226.5 & 3226.5 & 3226.5 & 3218.1 \\
\hline 1551.4 & 1754.9 & 3191.2 & 3236 & 3238.4 & 3241.7 & 3234 & 3234.1 & 3234 & 3224 \\
\hline 1842.8 & 2925.9 & 3232.2 & 3262.6 & 3255.3 & 3264.5 & 3255.9 & 3256 & 3255.9 & 3234.5 \\
\hline 3124.8 & 3078.8 & 3243.6 & 3388.5 & 3378.4 & 3389.5 & 3384.4 & 3384.5 & 3384.3 & 3236.3 \\
\hline 3128.1 & 3080.6 & & & & & & & & \\
\hline 3167.7 & 3184.4 & & & & & & & & \\
\hline 3222.1 & 3188.3 & & & & & & & & \\
\hline 3227.9 & 3189.6 & & & & & & & & \\
\hline 3236.2 & 3233.1 & & & & & & & & \\
\hline 3239.1 & 3237.0 & & & & & & & & \\
\hline
\end{tabular}


Table S2: Optimized geometries in Cartesian Coordinates of reactants, transition states and products for the reaction of isopropyl formate with $\mathrm{Cl}$ atoms at MP2/6-31+G (d, p) level of theory.

\begin{tabular}{|c|c|c|c|c|c|}
\hline \multicolumn{7}{|c|}{ Isopropyl formate } \\
\hline Center & Atomic & Atomic & \multicolumn{2}{|c|}{ Coordinates (Angstroms) } \\
\cline { 4 - 6 } number & number & Type & $\mathrm{X}$ & $\mathrm{Y}$ & $\mathrm{Z}$ \\
\hline 1 & 6 & 0 & 1.920368 & -0.80797 & -0.25848 \\
\hline 2 & 6 & 0 & 0.670925 & 0.045599 & -0.28064 \\
\hline 3 & 1 & 0 & 0.259865 & 0.110112 & -1.28858 \\
\hline 4 & 1 & 0 & 1.716283 & -1.80096 & -0.6556 \\
\hline 5 & 1 & 0 & 2.694991 & -0.34083 & -0.86703 \\
\hline 6 & 1 & 0 & 2.29153 & -0.90712 & 0.761362 \\
\hline 7 & 6 & 0 & 0.876662 & 1.434563 & 0.292169 \\
\hline 8 & 1 & 0 & -0.05028 & 2.004 & 0.268117 \\
\hline 9 & 1 & 0 & 1.229879 & 1.363373 & 1.321098 \\
\hline 10 & 1 & 0 & 1.624276 & 1.968048 & -0.29591 \\
\hline 11 & 8 & 0 & -0.30495 & -0.67144 & 0.54305 \\
\hline 12 & 6 & 0 & -1.60348 & -0.43088 & 0.272704 \\
\hline 13 & 8 & 0 & -2.03614 & 0.31889 & -0.58652 \\
\hline 14 & 1 & 0 & -2.22466 & -1.02407 & 0.949736 \\
\hline
\end{tabular}

\begin{tabular}{|c|c|c|c|c|c|}
\hline \multicolumn{7}{|c|}{ RC1 } \\
\hline Center & Atomic & Atomic & \multicolumn{2}{|c|}{ Coordinates (Angstroms) } \\
\cline { 4 - 6 } number & number & Type & $\mathrm{X}$ & $\mathrm{Y}$ & $\mathrm{Z}$ \\
\hline 1 & 6 & 0 & 2.319510 & 1.094459 & -0.831853 \\
\hline 2 & 6 & 0 & 1.139929 & 0.464365 & -0.1244 \\
\hline 3 & 1 & 0 & 0.232509 & 0.564908 & -0.71893 \\
\hline 4 & 1 & 0 & 2.452159 & 0.661077 & -1.82176 \\
\hline 5 & 1 & 0 & 2.150763 & 2.166076 & -0.9397 \\
\hline 6 & 1 & 0 & 3.23185 & 0.941808 & -0.25566 \\
\hline 7 & 6 & 0 & 0.917661 & 0.996172 & 1.277784 \\
\hline 8 & 1 & 0 & 0.082683 & 0.490179 & 1.758356 \\
\hline 9 & 1 & 0 & 1.818277 & 0.853122 & 1.875337 \\
\hline 10 & 1 & 0 & 0.692273 & 2.062131 & 1.234785 \\
\hline 11 & 8 & 0 & 1.453871 & -0.96694 & -0.04454 \\
\hline 12 & 6 & 0 & 0.409355 & -1.80709 & 0.037788 \\
\hline 13 & 8 & 0 & -0.77386 & -1.50149 & 0.045879 \\
\hline 14 & 1 & 0 & 0.776237 & -2.8355 & 0.093916 \\
\hline 15 & 17 & 0 & -2.68209 & 0.609191 & -0.19959 \\
\hline
\end{tabular}




\begin{tabular}{|c|c|c|c|c|c|}
\hline \multicolumn{7}{|c|}{ TS1 } \\
\hline Center & Atomic & Atomic & \multicolumn{2}{|c|}{ Coordinates (Angstroms) } \\
\cline { 4 - 6 } number & number & Type & $\mathrm{X}$ & $\mathrm{Y}$ & $\mathrm{Z}$ \\
\hline 1 & 6 & 0 & 0.327108 & -2.05686 & -0.63117 \\
\hline 2 & 6 & 0 & -0.20772 & -0.8285 & 0.045764 \\
\hline 3 & 1 & 0 & 0.814633 & 0.004438 & 0.031274 \\
\hline 4 & 1 & 0 & 0.644055 & -1.82725 & -1.64624 \\
\hline 5 & 1 & 0 & 1.173883 & -2.44875 & -0.07068 \\
\hline 6 & 1 & 0 & -0.45622 & -2.81795 & -0.6678 \\
\hline 7 & 6 & 0 & -0.58579 & -0.952 & 1.497823 \\
\hline 8 & 1 & 0 & -0.79394 & 0.013033 & 1.948524 \\
\hline 9 & 1 & 0 & -1.47298 & -1.58736 & 1.573736 \\
\hline 10 & 1 & 0 & 0.231812 & -1.42967 & 2.035184 \\
\hline 11 & 8 & 0 & -1.18395 & -0.25598 & -0.80182 \\
\hline 12 & 6 & 0 & -1.65965 & 1.002147 & -0.53579 \\
\hline 13 & 8 & 0 & -1.30437 & 1.725719 & 0.367937 \\
\hline 14 & 1 & 0 & -2.40949 & 1.242694 & -1.29268 \\
\hline 15 & 17 & 0 & 2.054772 & 0.829656 & -0.04118 \\
\hline
\end{tabular}

\begin{tabular}{|c|c|c|c|c|c|}
\hline \multicolumn{7}{|c|}{ TS2 } \\
\hline Center & Atomic & Atomic & \multicolumn{2}{|c|}{ Coordinates (Angstroms) } \\
\cline { 4 - 6 } number & number & Type & $\mathrm{X}$ & $\mathrm{Y}$ & $\mathrm{Z}$ \\
\hline 1 & 6 & 0 & 0.551865 & 1.553736 & 0.170165 \\
\hline 2 & 6 & 0 & -0.64344 & 0.734365 & -0.19876 \\
\hline 3 & 1 & 0 & -0.49469 & 0.240159 & -1.15964 \\
\hline 4 & 1 & 0 & 1.595985 & 0.64729 & 0.05852 \\
\hline 5 & 1 & 0 & 0.837186 & 2.32235 & -0.5414 \\
\hline 6 & 1 & 0 & 0.630645 & 1.83855 & 1.215046 \\
\hline 7 & 6 & 0 & -1.92842 & 1.55342 & -0.22702 \\
\hline 8 & 1 & 0 & -2.76585 & 0.904992 & -0.47951 \\
\hline 9 & 1 & 0 & -2.10491 & 2.010183 & 0.745931 \\
\hline 10 & 1 & 0 & -1.8563 & 2.33745 & -0.98071 \\
\hline 11 & 8 & 0 & -0.74133 & -0.28851 & 0.828607 \\
\hline 12 & 6 & 0 & -1.24863 & -1.4784 & 0.427659 \\
\hline 13 & 8 & 0 & -1.6655 & -1.73711 & -0.68631 \\
\hline 14 & 1 & 0 & -1.22695 & -2.17406 & 1.269596 \\
\hline 15 & 17 & 0 & 2.603015 & -0.35886 & -0.1352 \\
\hline
\end{tabular}




\begin{tabular}{|c|c|c|c|c|c|}
\hline \multicolumn{7}{|c|}{ TS3 } \\
\hline Center & Atomic & Atomic & \multicolumn{2}{|c|}{ Coordinates (Angstroms) } \\
\cline { 4 - 6 } number & number & Type & $\mathrm{X}$ & $\mathrm{Y}$ & $\mathrm{Z}$ \\
\hline 1 & 6 & 0 & 0.664891 & -1.15504 & 0.267439 \\
\hline 2 & 6 & 0 & -0.37104 & -0.07271 & 0.166122 \\
\hline 3 & 1 & 0 & -0.35543 & 0.383596 & -0.82395 \\
\hline 4 & 1 & 0 & 0.640232 & -1.91503 & -0.50654 \\
\hline 5 & 1 & 0 & 1.862227 & -0.50103 & -0.04911 \\
\hline 6 & 1 & 0 & 0.885472 & -1.52289 & 1.265102 \\
\hline 7 & 6 & 0 & -0.26345 & 0.983476 & 1.246452 \\
\hline 8 & 1 & 0 & -1.05567 & 1.720268 & 1.131295 \\
\hline 9 & 1 & 0 & -0.34268 & 0.519578 & 2.229576 \\
\hline 10 & 1 & 0 & 0.697151 & 1.492409 & 1.171713 \\
\hline 11 & 8 & 0 & -1.64247 & -0.78995 & 0.306576 \\
\hline 12 & 6 & 0 & -2.703 & -0.22877 & -0.3246 \\
\hline 13 & 8 & 0 & -2.68545 & 0.796292 & -0.98004 \\
\hline 14 & 1 & 0 & -3.58544 & -0.85 & -0.1506 \\
\hline 15 & 17 & 0 & 3.05371 & 0.203566 & -0.41249 \\
\hline
\end{tabular}

\begin{tabular}{|c|c|c|c|c|c|}
\hline \multicolumn{7}{|c|}{ TS4 } \\
\hline Center & Atomic & Atomic & \multicolumn{2}{|c|}{ Coordinates (Angstroms) } \\
\cline { 4 - 6 } number & number & Type & $\mathrm{X}$ & $\mathrm{Y}$ & $\mathrm{Z}$ \\
\hline 1 & 6 & 0 & -0.67783 & 0.703583 & 1.248261 \\
\hline 2 & 6 & 0 & 0.535441 & 0.688983 & 0.374684 \\
\hline 3 & 1 & 0 & 1.380367 & 1.104284 & 0.936515 \\
\hline 4 & 1 & 0 & -0.65769 & 0.039751 & 2.106559 \\
\hline 5 & 1 & 0 & -1.11178 & 1.681441 & 1.432933 \\
\hline 6 & 1 & 0 & -1.67499 & 0.090807 & 0.491225 \\
\hline 7 & 6 & 0 & 0.376156 & 1.432449 & -0.93934 \\
\hline 8 & 1 & 0 & 1.310303 & 1.407154 & -1.49605 \\
\hline 9 & 1 & 0 & -0.41475 & 0.978103 & -1.53317 \\
\hline 10 & 1 & 0 & 0.118573 & 2.473801 & -0.74368 \\
\hline 11 & 8 & 0 & 0.82013 & -0.71553 & 0.121197 \\
\hline 12 & 6 & 0 & 2.119761 & -1.02079 & -0.09542 \\
\hline 13 & 8 & 0 & 3.050124 & -0.23451 & -0.08151 \\
\hline 14 & 1 & 0 & 2.205627 & -2.09391 & -0.2821 \\
\hline 15 & 17 & 0 & -2.71993 & -0.52391 & -0.27993 \\
\hline
\end{tabular}




\begin{tabular}{|c|c|c|c|c|c|}
\hline \multicolumn{7}{|c|}{ TS5 } \\
\hline Center & Atomic & Atomic & \multicolumn{2}{|c|}{ Coordinates (Angstroms) } \\
\cline { 4 - 6 } number & number & Type & $\mathrm{X}$ & $\mathrm{Y}$ & $\mathrm{Z}$ \\
\hline 1 & 6 & 0 & 1.928793 & -1.55307 & 0.226229 \\
\hline 2 & 6 & 0 & 0.643555 & -0.73439 & 0.198872 \\
\hline 3 & 1 & 0 & 0.495207 & -0.24042 & 1.159935 \\
\hline 4 & 1 & 0 & 2.76601 & -0.90472 & 0.479622 \\
\hline 5 & 1 & 0 & 1.856854 & -2.33814 & 0.978849 \\
\hline 6 & 1 & 0 & 2.105476 & -2.00848 & -0.74732 \\
\hline 7 & 6 & 0 & -0.55174 & -1.554 & -0.16955 \\
\hline 8 & 1 & 0 & -1.59578 & -0.64712 & -0.05808 \\
\hline 9 & 1 & 0 & -0.63053 & -1.83944 & -1.21425 \\
\hline 10 & 1 & 0 & -0.83701 & -2.32222 & 0.542454 \\
\hline 11 & 8 & 0 & 0.740464 & 0.288735 & -0.82835 \\
\hline 12 & 6 & 0 & 1.248054 & 1.478523 & -0.42748 \\
\hline 13 & 8 & 0 & 1.666092 & 1.736822 & 0.686154 \\
\hline 14 & 1 & 0 & 1.225375 & 2.174527 & -1.2691 \\
\hline 15 & 17 & 0 & -2.60294 & 0.358777 & 0.135121 \\
\hline
\end{tabular}

\begin{tabular}{|c|c|c|c|c|c|}
\hline \multicolumn{7}{|c|}{ TS6 } \\
\hline Center & Atomic & Atomic & \multicolumn{2}{|c|}{ Coordinates (Angstroms) } \\
\cline { 4 - 6 } number & number & Type & $\mathrm{X}$ & $\mathrm{Y}$ & $\mathrm{Z}$ \\
\hline 1 & 6 & 0 & 0.041595 & 2.133566 & -0.36945 \\
\hline 2 & 6 & 0 & -0.6186 & 0.927305 & 0.265121 \\
\hline 3 & 1 & 0 & -1.5651 & 1.207243 & 0.742367 \\
\hline 4 & 1 & 0 & -0.63515 & 2.594313 & -1.08612 \\
\hline 5 & 1 & 0 & 0.286981 & 2.864653 & 0.4009 \\
\hline 6 & 1 & 0 & 0.955029 & 1.839181 & -0.88266 \\
\hline 7 & 6 & 0 & 0.226243 & 0.232086 & 1.291948 \\
\hline 8 & 1 & 0 & -0.16381 & -0.69566 & 1.695813 \\
\hline 9 & 1 & 0 & 1.322729 & -0.24431 & 0.567328 \\
\hline 10 & 1 & 0 & 0.706185 & 0.879817 & 2.019496 \\
\hline 11 & 8 & 0 & -0.92603 & 0.014939 & -0.82631 \\
\hline 12 & 6 & 0 & -1.86513 & -0.92182 & -0.56932 \\
\hline 13 & 8 & 0 & -2.45975 & -1.06432 & 0.485157 \\
\hline 14 & 1 & 0 & -2.01168 & -1.53487 & -1.46169 \\
\hline 15 & 17 & 0 & 2.44038 & -0.74954 & -0.17506 \\
\hline
\end{tabular}




\begin{tabular}{|c|c|c|c|c|c|}
\hline \multicolumn{7}{|c|}{ TS7 } \\
\hline Center & Atomic & Atomic & \multicolumn{2}{|c|}{ Coordinates (Angstroms) } \\
\cline { 4 - 6 } number & number & Type & $\mathrm{X}$ & $\mathrm{Y}$ & $\mathrm{Z}$ \\
\hline 1 & 6 & 0 & -0.06419 & 1.952545 & -0.29864 \\
\hline 2 & 6 & 0 & 0.416289 & 0.527885 & -0.14115 \\
\hline 3 & 1 & 0 & 0.370571 & -0.01783 & -1.08381 \\
\hline 4 & 1 & 0 & 0.517623 & 2.46209 & -1.06419 \\
\hline 5 & 1 & 0 & -1.11301 & 1.956217 & -0.59219 \\
\hline 6 & 1 & 0 & 0.042066 & 2.492099 & 0.641981 \\
\hline 7 & 6 & 0 & -0.29907 & -0.24095 & 0.934588 \\
\hline 8 & 1 & 0 & -0.02489 & -1.28457 & 1.043985 \\
\hline 9 & 1 & 0 & -0.48089 & 0.295155 & 1.861694 \\
\hline 10 & 1 & 0 & -1.59242 & -0.37709 & 0.43338 \\
\hline 11 & 8 & 0 & 1.828232 & 0.621972 & 0.250931 \\
\hline 12 & 6 & 0 & 2.580819 & -0.47509 & -0.00214 \\
\hline 13 & 8 & 0 & 2.181863 & -1.51882 & -0.48614 \\
\hline 14 & 1 & 0 & 3.612712 & -0.2749 & 0.297905 \\
\hline 15 & 17 & 0 & -2.89504 & -0.50958 & -0.15371 \\
\hline
\end{tabular}

\begin{tabular}{|c|c|c|c|c|c|}
\hline \multicolumn{6}{|c|}{ TS8 } \\
\hline Center & Atomic & Atomic & \multicolumn{2}{|c|}{ Coordinates (Angstroms) } \\
\cline { 4 - 6 } number & number & Type & $\mathrm{X}$ & $\mathrm{Y}$ & $\mathrm{Z}$ \\
\hline 1 & 6 & 0 & 2.415977 & -1.40409 & -0.44825 \\
\hline 2 & 6 & 0 & 1.775772 & -0.05758 & -0.20595 \\
\hline 3 & 1 & 0 & 1.862343 & 0.587679 & -1.08094 \\
\hline 4 & 1 & 0 & 1.997064 & -1.87844 & -1.33353 \\
\hline 5 & 1 & 0 & 3.487923 & -1.27161 & -0.5956 \\
\hline 6 & 1 & 0 & 2.259387 & -2.05571 & 0.41043 \\
\hline 7 & 6 & 0 & 2.264689 & 0.648283 & 1.040408 \\
\hline 8 & 1 & 0 & 1.754957 & 1.599889 & 1.179457 \\
\hline 9 & 1 & 0 & 2.099207 & 0.01855 & 1.913938 \\
\hline 10 & 1 & 0 & 3.33351 & 0.843483 & 0.948525 \\
\hline 11 & 8 & 0 & 0.327499 & -0.35189 & -0.04235 \\
\hline 12 & 6 & 0 & -0.49523 & 0.648966 & -0.29848 \\
\hline 13 & 8 & 0 & -0.29344 & 1.780755 & -0.66317 \\
\hline 14 & 1 & 0 & -1.63711 & 0.222817 & -0.09605 \\
\hline 15 & 17 & 0 & -3.01159 & -0.50065 & 0.221857 \\
\hline
\end{tabular}




\begin{tabular}{|c|c|c|c|c|c|}
\hline \multicolumn{7}{|c|}{ PC1 } \\
\hline Center & Atomic & Atomic & \multicolumn{2}{|c|}{ Coordinates (Angstroms) } \\
\cline { 4 - 6 } number & number & Type & $\mathrm{X}$ & $\mathrm{Y}$ & $\mathrm{Z}$ \\
\hline 1 & 6 & 0 & 1.264307 & 1.744831 & -0.90778 \\
\hline 2 & 6 & 0 & 0.965035 & 0.636866 & 0.033374 \\
\hline 3 & 1 & 0 & -1.59881 & -0.58995 & -0.05198 \\
\hline 4 & 1 & 0 & 1.134268 & 1.421963 & -1.93976 \\
\hline 5 & 1 & 0 & 0.593694 & 2.580189 & -0.71337 \\
\hline 6 & 1 & 0 & 2.29584 & 2.097428 & -0.78966 \\
\hline 7 & 6 & 0 & 0.782241 & 0.857393 & 1.494295 \\
\hline 8 & 1 & 0 & 0.361845 & -0.02191 & 1.979824 \\
\hline 9 & 1 & 0 & 1.742012 & 1.088367 & 1.971794 \\
\hline 10 & 1 & 0 & 0.108056 & 1.696409 & 1.657093 \\
\hline 11 & 8 & 0 & 1.710773 & -0.53323 & -0.29281 \\
\hline 12 & 6 & 0 & 1.062377 & -1.71101 & -0.21026 \\
\hline 13 & 8 & 0 & -0.11808 & -1.87021 & 0.0612 \\
\hline 14 & 1 & 0 & 1.756283 & -2.52442 & -0.43088 \\
\hline 15 & 17 & 0 & -2.56344 & 0.25359 & -0.13459 \\
\hline
\end{tabular}

\begin{tabular}{|c|c|c|c|c|c|}
\hline \multicolumn{6}{|c|}{ P1 } \\
\hline Center & Atomic & Atomic & \multicolumn{2}{|c|}{ Coordinates (Angstroms) } \\
\cline { 4 - 6 } number & number & Type & $\mathrm{X}$ & $\mathrm{Y}$ & $\mathrm{Z}$ \\
\hline 1 & 6 & 0 & 1.97844 & -0.73239 & -0.30644 \\
\hline 2 & 6 & 0 & 0.719503 & 0.043759 & -0.14822 \\
\hline 3 & 1 & 0 & 1.785538 & -1.68785 & -0.79188 \\
\hline 4 & 1 & 0 & 2.690725 & -0.16775 & -0.90609 \\
\hline 5 & 1 & 0 & 2.440596 & -0.93578 & 0.667186 \\
\hline 6 & 6 & 0 & 0.739089 & 1.480389 & 0.249692 \\
\hline 7 & 1 & 0 & -0.22123 & 1.957532 & 0.070486 \\
\hline 8 & 1 & 0 & 0.984363 & 1.578185 & 1.314452 \\
\hline 9 & 1 & 0 & 1.502705 & 2.006541 & -0.32113 \\
\hline 10 & 8 & 0 & -0.26986 & -0.752 & 0.479356 \\
\hline 11 & 6 & 0 & -1.57531 & -0.49731 & 0.199305 \\
\hline 12 & 8 & 0 & -2.00022 & 0.334734 & -0.57797 \\
\hline 13 & 1 & 0 & -2.19238 & -1.17945 & 0.78985 \\
\hline
\end{tabular}




\begin{tabular}{|c|c|c|c|c|c|}
\hline \multicolumn{6}{|c|}{ P2 } \\
\hline Center & Atomic & Atomic & \multicolumn{2}{|c|}{ Coordinates (Angstroms) } \\
\cline { 4 - 6 } number & number & Type & $\mathrm{X}$ & $\mathrm{Y}$ & $\mathrm{Z}$ \\
\hline 1 & 6 & 0 & 1.913207 & -0.86359 & -0.32247 \\
\hline 2 & 6 & 0 & 0.711899 & 0.008424 & -0.28585 \\
\hline 3 & 1 & 0 & 0.295241 & 0.143669 & -1.28603 \\
\hline 4 & 1 & 0 & 2.692469 & -0.65962 & -1.03997 \\
\hline 5 & 1 & 0 & 2.108924 & -1.54195 & 0.492596 \\
\hline 6 & 6 & 0 & 0.964346 & 1.375088 & 0.345766 \\
\hline 7 & 1 & 0 & 0.04932 & 1.965067 & 0.344944 \\
\hline 8 & 1 & 0 & 1.319646 & 1.24954 & 1.367688 \\
\hline 9 & 1 & 0 & 1.724061 & 1.907964 & -0.22594 \\
\hline 10 & 8 & 0 & -0.28098 & -0.70522 & 0.516982 \\
\hline 11 & 6 & 0 & -1.57191 & -0.41778 & 0.25367 \\
\hline 12 & 8 & 0 & -1.9796 & 0.372299 & -0.58086 \\
\hline 13 & 1 & 0 & -2.21028 & -1.0142 & 0.911093 \\
\hline
\end{tabular}

\begin{tabular}{|c|c|c|c|c|c|}
\hline \multicolumn{6}{|c|}{ P3 } \\
\hline Center & Atomic & Atomic & \multicolumn{2}{|c|}{ Coordinates (Angstroms) } \\
\cline { 4 - 6 } number & number & Type & $\mathrm{X}$ & $\mathrm{Y}$ & $\mathrm{Z}$ \\
\hline 1 & 6 & 0 & 1.907201 & -0.88414 & -0.30193 \\
\hline 2 & 6 & 0 & 0.716546 & 0.003027 & -0.29376 \\
\hline 3 & 1 & 0 & 0.266905 & 0.060124 & -1.28559 \\
\hline 4 & 1 & 0 & 1.884311 & -1.81128 & -0.8529 \\
\hline 5 & 1 & 0 & 2.71195 & -0.71872 & 0.398303 \\
\hline 6 & 6 & 0 & 0.993667 & 1.393402 & 0.244392 \\
\hline 7 & 1 & 0 & 0.086319 & 1.993733 & 0.238284 \\
\hline 8 & 1 & 0 & 1.376484 & 1.328231 & 1.262746 \\
\hline 9 & 1 & 0 & 1.740375 & 1.884979 & -0.37952 \\
\hline 10 & 8 & 0 & -0.28016 & -0.65399 & 0.580924 \\
\hline 11 & 6 & 0 & -1.57259 & -0.38775 & 0.30043 \\
\hline 12 & 8 & 0 & -1.98565 & 0.334875 & -0.59106 \\
\hline 13 & 1 & 0 & -2.20876 & -0.93142 & 1.004919 \\
\hline
\end{tabular}




\begin{tabular}{|c|c|c|c|c|c|}
\hline \multicolumn{6}{|c|}{ P4 } \\
\hline Center & Atomic & Atomic & \multicolumn{2}{|c|}{ Coordinates (Angstroms) } \\
\cline { 4 - 6 } number & number & Type & $\mathrm{X}$ & $\mathrm{Y}$ & $\mathrm{Z}$ \\
\hline 1 & 6 & 0 & 1.913587 & -0.87741 & -0.2757 \\
\hline 2 & 6 & 0 & 0.716361 & 0.000254 & -0.27231 \\
\hline 3 & 1 & 0 & 0.290845 & 0.099312 & -1.27687 \\
\hline 4 & 1 & 0 & 1.796573 & -1.94876 & -0.24817 \\
\hline 5 & 1 & 0 & 2.88275 & -0.45665 & -0.4929 \\
\hline 6 & 6 & 0 & 0.976225 & 1.382466 & 0.305101 \\
\hline 7 & 1 & 0 & 0.069419 & 1.982767 & 0.275491 \\
\hline 8 & 1 & 0 & 1.323756 & 1.295344 & 1.333587 \\
\hline 9 & 1 & 0 & 1.744113 & 1.885628 & -0.2832 \\
\hline 10 & 8 & 0 & -0.28689 & -0.68848 & 0.543913 \\
\hline 11 & 6 & 0 & -1.5746 & -0.3987 & 0.265232 \\
\hline 12 & 8 & 0 & -1.9726 & 0.373075 & -0.59087 \\
\hline 13 & 1 & 0 & -2.22101 & -0.97403 & 0.933764 \\
\hline
\end{tabular}

\begin{tabular}{|c|c|c|c|c|c|}
\hline \multicolumn{7}{|c|}{ P5 } \\
\hline Center & Atomic & Atomic & \multicolumn{2}{|c|}{ Coordinates (Angstroms) } \\
\cline { 4 - 6 } number & number & Type & $\mathrm{X}$ & $\mathrm{Y}$ & $\mathrm{Z}$ \\
\hline 1 & 6 & 0 & 2.012653 & -0.63226 & -0.2429 \\
\hline 2 & 6 & 0 & 0.696694 & 0.114981 & -0.28825 \\
\hline 3 & 1 & 0 & 0.308417 & 0.172566 & -1.30531 \\
\hline 4 & 1 & 0 & 1.900796 & -1.62833 & -0.66735 \\
\hline 5 & 1 & 0 & 2.76224 & -0.08652 & -0.8153 \\
\hline 6 & 1 & 0 & 2.357713 & -0.72496 & 0.786381 \\
\hline 7 & 6 & 0 & 0.755644 & 1.469097 & 0.320082 \\
\hline 8 & 1 & 0 & 1.411093 & 1.646451 & 1.159954 \\
\hline 9 & 1 & 0 & 0.026093 & 2.217209 & 0.055624 \\
\hline 10 & 8 & 0 & -0.24758 & -0.73722 & 0.473969 \\
\hline 11 & 6 & 0 & -1.55421 & -0.49631 & 0.247332 \\
\hline 12 & 8 & 0 & -2.01188 & 0.343892 & -0.50924 \\
\hline 13 & 1 & 0 & -2.15538 & -1.18279 & 0.8506 \\
\hline
\end{tabular}




\begin{tabular}{|c|c|c|c|c|c|}
\hline \multicolumn{6}{|c|}{ P6 } \\
\hline Center & Atomic & Atomic & \multicolumn{2}{|c|}{ Coordinates (Angstroms) } \\
\cline { 4 - 6 } number & number & Type & $\mathrm{X}$ & $\mathrm{Y}$ & $\mathrm{Z}$ \\
\hline 1 & 6 & 0 & -2.01303 & -0.63166 & 0.242686 \\
\hline 2 & 6 & 0 & -0.69655 & 0.114933 & 0.28829 \\
\hline 3 & 1 & 0 & -0.30868 & 0.172332 & 1.305499 \\
\hline 4 & 1 & 0 & -1.90188 & -1.62774 & 0.667293 \\
\hline 5 & 1 & 0 & -2.76223 & -0.08527 & 0.814985 \\
\hline 6 & 1 & 0 & -2.35788 & -0.72423 & -0.78666 \\
\hline 7 & 6 & 0 & -0.75511 & 1.468959 & -0.3203 \\
\hline 8 & 1 & 0 & -0.02602 & 2.217286 & -0.05522 \\
\hline 9 & 1 & 0 & -1.40977 & 1.645878 & -1.16087 \\
\hline 10 & 8 & 0 & 0.247513 & -0.73791 & -0.4734 \\
\hline 11 & 6 & 0 & 1.554239 & -0.49652 & -0.2471 \\
\hline 12 & 8 & 0 & 2.011708 & 0.344267 & 0.508845 \\
\hline 13 & 1 & 0 & 2.155365 & -1.18334 & -0.85003 \\
\hline
\end{tabular}

\begin{tabular}{|c|c|c|c|c|c|}
\hline \multicolumn{6}{|c|}{ P7 } \\
\hline Center & Atomic & Atomic & \multicolumn{2}{|c|}{ Coordinates (Angstroms) } \\
\cline { 4 - 6 } number & number & Type & $\mathrm{X}$ & $\mathrm{Y}$ & $\mathrm{Z}$ \\
\hline 1 & 6 & 0 & 2.012549 & -0.63241 & -0.24305 \\
\hline 2 & 6 & 0 & 0.696598 & 0.114917 & -0.28821 \\
\hline 3 & 1 & 0 & 0.308232 & 0.172543 & -1.30522 \\
\hline 4 & 1 & 0 & 1.900577 & -1.62851 & -0.6674 \\
\hline 5 & 1 & 0 & 2.761958 & -0.08669 & -0.81571 \\
\hline 6 & 1 & 0 & 2.357835 & -0.72502 & 0.786157 \\
\hline 7 & 6 & 0 & 0.755893 & 1.469024 & 0.320128 \\
\hline 8 & 1 & 0 & 0.027361 & 2.217831 & 0.054808 \\
\hline 9 & 1 & 0 & 1.410444 & 1.645899 & 1.160809 \\
\hline 10 & 8 & 0 & -0.2476 & -0.73701 & 0.474206 \\
\hline 11 & 6 & 0 & -1.55427 & -0.4962 & 0.247372 \\
\hline 12 & 8 & 0 & -2.01186 & 0.343825 & -0.50941 \\
\hline 13 & 1 & 0 & -2.1554 & -1.1826 & 0.850748 \\
\hline
\end{tabular}




\begin{tabular}{|c|c|c|c|c|c|}
\hline \multicolumn{7}{|c|}{ P8 } \\
\hline Center & Atomic & Atomic & \multicolumn{2}{|c|}{ Coordinates (Angstroms) } \\
\cline { 4 - 6 } number & number & Type & $\mathrm{X}$ & $\mathrm{Y}$ & $\mathrm{Z}$ \\
\hline 1 & 6 & 0 & 1.913116 & -0.7675 & -0.23752 \\
\hline 2 & 6 & 0 & 0.636448 & 0.038729 & -0.28892 \\
\hline 3 & 1 & 0 & 0.202543 & 0.029394 & -1.28994 \\
\hline 4 & 1 & 0 & 1.741926 & -1.78523 & -0.58315 \\
\hline 5 & 1 & 0 & 2.663706 & -0.30181 & -0.8768 \\
\hline 6 & 1 & 0 & 2.296102 & -0.80059 & 0.781887 \\
\hline 7 & 6 & 0 & 0.782581 & 1.456872 & 0.220497 \\
\hline 8 & 1 & 0 & -0.16897 & 1.984231 & 0.188341 \\
\hline 9 & 1 & 0 & 1.15404 & 1.447516 & 1.244869 \\
\hline 10 & 1 & 0 & 1.495102 & 1.99672 & -0.40433 \\
\hline 11 & 8 & 0 & -0.3159 & -0.68672 & 0.589401 \\
\hline 12 & 6 & 0 & -1.60416 & -0.48583 & 0.340921 \\
\hline 13 & 8 & 0 & -2.15315 & 0.183737 & -0.49824 \\
\hline
\end{tabular}

\begin{tabular}{|c|c|c|c|c|c|}
\hline \multicolumn{7}{|c|}{ HCl } \\
\hline Center & Atomic & Atomic & \multicolumn{2}{|c|}{ Coordinates (Angstroms) } \\
\cline { 4 - 6 } Number & Number & Type & $\mathrm{X}$ & $\mathrm{Y}$ & $\mathrm{Z}$ \\
\hline 1 & 17 & 0 & 0 & 0 & 0.070556 \\
\hline 2 & 1 & 0 & 0 & 0 & -1.19946 \\
\hline
\end{tabular}

Table S3: Vibrational frequencies of reactants, reactive complexes, transition states, and products for the reaction of isobutyl formate with $\mathrm{Cl}$ atoms at MP2/6-31+G (d, p) level of theory.

\begin{tabular}{|l|l|l|l|l|l|l|l|l|}
\hline R1 & RC1 & RC2 & RC3 & TS1 & TS2 & TS3 & TS4 & TS5 \\
\hline
\end{tabular}




\begin{tabular}{|c|c|c|c|c|c|c|c|c|}
\hline 43.5 & 26.7 & 26.5 & 14.3 & -879.7 & -992.4 & -997.1 & -1096.7 & -1041.9 \\
\hline 107.8 & 47.9 & 47.9 & 35.5 & 22.9 & 42.5 & 47.4 & 30.8 & 28.1 \\
\hline 156.2 & 58.3 & 58.3 & 44.0 & 68.6 & 59.0 & 65.5 & 39.7 & 50.0 \\
\hline 219.9 & 86.7 & 86.7 & 80.4 & 109.7 & 88.9 & 91.8 & 94.8 & 103.6 \\
\hline 254.0 & 112.0 & 111.9 & 110.9 & 130.9 & 135.0 & 135.9 & 112.4 & 128.7 \\
\hline 269.3 & 162.1 & 162.1 & 164.3 & 162.3 & 165.5 & 159.7 & 159.5 & 156.4 \\
\hline 340.5 & 218.6 & 218.6 & 219.5 & 200.9 & 215.3 & 213.4 & 218.7 & 219.7 \\
\hline 365.1 & 253.2 & 253.2 & 253.8 & 222.6 & 249.0 & 240.6 & 236.0 & 268.4 \\
\hline 437.0 & 270.6 & 270.6 & 268.9 & 258.1 & 267.5 & 267.6 & 339.2 & 339.1 \\
\hline 456.9 & 336.5 & 336.5 & 343.7 & 274.9 & 317.3 & 310.6 & 362.6 & 363.9 \\
\hline 767.0 & 364.9 & 364.9 & 364.6 & 342.9 & 366.7 & 369.2 & 407.3 & 432.9 \\
\hline 878.7 & 438.8 & 438.7 & 437.5 & 370.7 & 384.5 & 440.2 & 424.2 & 444.8 \\
\hline 932.5 & 456.8 & 456.8 & 456.6 & 461.2 & 460.4 & 473.3 & 462.6 & 467.5 \\
\hline 952.1 & 764.4 & 764.4 & 766.8 & 569.2 & 545.9 & 502.6 & 558.0 & 544.1 \\
\hline 969.1 & 876.3 & 876.3 & 878.7 & 771.8 & 761.0 & 752.1 & 768.9 & 765.2 \\
\hline 1007.8 & 932.4 & 932.4 & 932.3 & 884.4 & 874.7 & 876.2 & 882.3 & 877.6 \\
\hline 1016.3 & 951.9 & 951.9 & 952.2 & 917.1 & 929.2 & 915.4 & 891.0 & 884.3 \\
\hline 1041.0 & 965.1 & 965.2 & 967.5 & 937.4 & 958.4 & 955.4 & 941.5 & 926.3 \\
\hline 1186.4 & 1006.7 & 1006.7 & 1007.2 & 982.7 & 975.5 & 988.1 & 945.0 & 966.4 \\
\hline 1211.5 & 1014.0 & 1014.1 & 1015.5 & 1005.3 & 1000.0 & 1011.3 & 980.3 & 998.6 \\
\hline 1230.3 & 1038.4 & 1038.4 & 1042.8 & 1025.4 & 1027.9 & 1027.0 & 1006.0 & 1010.4 \\
\hline 1236.6 & 1185.8 & 1185.8 & 1186.1 & 1039.2 & 1042.6 & 1039.6 & 1010.8 & 1015.0 \\
\hline 1296.0 & 1202.1 & 1202.0 & 1210.8 & 1057.2 & 1101.3 & 1097.0 & 1039.8 & 1038.8 \\
\hline 1353.4 & 1219.2 & 1219.2 & 1234.6 & 1061.9 & 1163.8 & 1126.6 & 1140.6 & 1075.7 \\
\hline 1402.9 & 1236.0 & 1236.0 & 1243.4 & 1203.0 & 1177.3 & 1174.5 & 1193.4 & 1195.9 \\
\hline 1420.9 & 1297.0 & 1297.0 & 1297.6 & 1220.0 & 1206.4 & 1190.8 & 1207.9 & 1212.2 \\
\hline 1441.1 & 1355.5 & 1355.6 & 1353.9 & 1262.4 & 1228.4 & 1237.0 & 1220.6 & 1219.9 \\
\hline 1443.2 & 1403.5 & 1403.5 & 1403.3 & 1296.0 & 1245.7 & 1269.6 & 1240.4 & 1259.8 \\
\hline 1463.4 & 1421.5 & 1421.5 & 1421.7 & 1335.7 & 1341.9 & 1334.9 & 1281.4 & 1284.4 \\
\hline 1529.6 & 1440.3 & 1440.3 & 1442.1 & 1416.1 & 1370.3 & 1373.0 & 1347.7 & 1334.5 \\
\hline 1533.4 & 1442.9 & 1442.9 & 1443.9 & 1424.3 & 1418.8 & 1413.6 & 1408.9 & 1380.2 \\
\hline 1545.4 & 1463.5 & 1463.5 & 1464.1 & 1437.2 & 1428.7 & 1420.8 & 1418.3 & 1419.0 \\
\hline 1549.4 & 1528.9 & 1528.9 & 1529.3 & 1456.0 & 1440.9 & 1444.0 & 1425.1 & 1437.6 \\
\hline 1555.4 & 1532.7 & 1532.7 & 1533.2 & 1510.3 & 1462.5 & 1466.4 & 1455.6 & 1455.8 \\
\hline 1769.5 & 1544.3 & 1544.3 & 1544.9 & 1519.9 & 1530.1 & 1531.3 & 1504.5 & 1500.8 \\
\hline 3106.7 & 1547.7 & 1547.6 & 1548.6 & 1528.9 & 1533.9 & 1534.6 & 1537.7 & 1541.0 \\
\hline 3108.2 & 1554.2 & 1554.2 & 1555.2 & 1533.3 & 1544.4 & 1547.3 & 1545.8 & 1545.2 \\
\hline 3117.1 & 1772.9 & 1772.9 & 1762.0 & 1543.1 & 1556.0 & 1555.5 & 1548.9 & 1551.9 \\
\hline 3126.7 & 3106.6 & 3106.6 & 3107.2 & 1773.1 & 1787.9 & 1788.4 & 1772.7 & 1774.4 \\
\hline 3165.3 & 3108.1 & 3108.1 & 3108.6 & 3091.3 & 3112.0 & 3061.2 & 3115.5 & 3053.2 \\
\hline 3192.8 & 3124.9 & 3124.9 & 3117.6 & 3092.7 & 3115.4 & 3116.8 & 3126.1 & 3120.1 \\
\hline
\end{tabular}




\begin{tabular}{|l|l|l|l|l|l|l|l|l|}
3196.3 & 3131.7 & 3131.7 & 3130.5 & 3097.6 & 3130.6 & 3122.3 & 3132.7 & 3141.6 \\
\hline 3201.3 & 3171.7 & 3171.8 & 3170.9 & 3180.2 & 3183.8 & 3193.7 & 3174.0 & 3171.5 \\
\hline 3210.7 & 3195.6 & 3195.8 & 3196.9 & 3185.7 & 3198.0 & 3198.6 & 3180.3 & 3189.5 \\
\hline 3222.3 & 3196.4 & 3196.4 & 3198.2 & 3191.3 & 3205.1 & 3212.3 & 3199.0 & 3209.9 \\
\hline & 3202.4 & 3202.5 & 3204.2 & 3194.4 & 3209.4 & 3216.4 & 3208.6 & 3217.8 \\
\hline & 3211.3 & 3211.3 & 3211.3 & 3227.5 & 3225.8 & 3219.9 & 3229.5 & 3220.7 \\
\hline & 3222.6 & 3222.6 & 3221.6 & 3242.5 & 3227.5 & 3229.2 & 3292.9 & 3297.6 \\
\hline
\end{tabular}

\begin{tabular}{|c|c|c|c|c|c|c|c|c|}
\hline TS6 & TS7 & TS8 & TS9 & TS10 & PC1 & PC2 & PC3 & P1 \\
\hline-1252.1 & -1096.0 & -1164.7 & -1095.2 & -1100.0 & 12.4 & 26.5 & 19.6 & 41.3 \\
\hline 44.0 & 33.4 & 29.5 & 30.5 & 32.3 & 35.8 & 40.7 & 37.3 & 66.3 \\
\hline 52.3 & 56.4 & 49.3 & 62.3 & 45.9 & 46.6 & 50.7 & 56.5 & 134.2 \\
\hline 77.0 & 75.0 & 76.7 & 68.5 & 58.3 & 68.6 & 79.6 & 79.3 & 148.1 \\
\hline 119.8 & 116.1 & 107.2 & 118.2 & 138.6 & 86.9 & 117.4 & 123.0 & 168.7 \\
\hline 156.4 & 162.4 & 145.9 & 162.0 & 163.7 & 141.3 & 156.9 & 163.3 & 262.7 \\
\hline 232.1 & 226.9 & 230.9 & 235.5 & 221.6 & 155.7 & 218.3 & 221.4 & 307.2 \\
\hline 253.7 & 264.7 & 270.7 & 264.4 & 254.6 & 177.2 & 257.8 & 258.2 & 339.4 \\
\hline 316.9 & 339.2 & 321.8 & 321.9 & 265.6 & 264.3 & 280.8 & 266.7 & 377.2 \\
\hline 341.5 & 365.5 & 338.9 & 336.6 & 347.2 & 309.3 & 309.8 & 298.7 & 461.6 \\
\hline 414.4 & 426.8 & 417.7 & 418.3 & 375.9 & 341.0 & 355.8 & 342.9 & 748.6 \\
\hline 424.1 & 446.2 & 435.5 & 440.0 & 397.2 & 362.0 & 374.4 & 365.8 & 862.7 \\
\hline 480.2 & 477.3 & 454.0 & 451.7 & 434.6 & 379.3 & 398.9 & 392.5 & 940.3 \\
\hline 523.2 & 530.7 & 556.1 & 563.4 & 457.9 & 433.5 & 427.0 & 452.6 & 958.7 \\
\hline 771.0 & 765.4 & 769.4 & 768.0 & 788.6 & 464.5 & 468.5 & 472.9 & 970.9 \\
\hline 888.1 & 870.6 & 874.2 & 869.1 & 879.1 & 749.8 & 713.3 & 700.7 & 1024.4 \\
\hline 922.7 & 898.6 & 899.4 & 903.3 & 921.8 & 862.7 & 763.8 & 772.5 & 1040.8 \\
\hline 935.0 & 931.7 & 940.8 & 934.4 & 947.2 & 940.3 & 887.0 & 879.5 & 1044.3 \\
\hline 971.1 & 965.2 & 951.9 & 951.9 & 960.3 & 954.7 & 954.8 & 952.2 & 1113.9 \\
\hline 993.5 & 996.3 & 976.9 & 994.0 & 962.1 & 971.6 & 967.9 & 985.5 & 1227.0 \\
\hline 1011.4 & 1015.3 & 1003.2 & 997.0 & 1002.7 & 1024.5 & 1000.3 & 1010.6 & 1259.8 \\
\hline 1043.0 & 1016.9 & 1010.4 & 1014.6 & 1012.2 & 1039.3 & 1020.0 & 1017.7 & 1311.5 \\
\hline 1051.6 & 1039.7 & 1039.6 & 1039.8 & 1183.5 & 1043.9 & 1072.5 & 1072.7 & 1351.8 \\
\hline 1107.2 & 1064.4 & 1148.4 & 1137.7 & 1205.5 & 1122.2 & 1148.3 & 1142.2 & 1419.3 \\
\hline 1196.5 & 1203.1 & 1185.0 & 1184.5 & 1226.2 & 1218.2 & 1195.2 & 1189.0 & 1433.8 \\
\hline 1212.2 & 1216.1 & 1209.0 & 1210.0 & 1235.2 & 1255.7 & 1210.7 & 1196.9 & 1446.8 \\
\hline 1222.4 & 1225.0 & 1219.6 & 1224.8 & 1240.8 & 1308.5 & 1235.4 & 1244.5 & 1459.9 \\
\hline 1230.1 & 1252.0 & 1239.6 & 1236.3 & 1289.0 & 1348.7 & 1332.0 & 1338.9 & 1516.4 \\
\hline 1293.9 & 1296.5 & 1293.9 & 1298.6 & 1353.7 & 1416.4 & 1366.2 & 1370.4 & 1520.0 \\
\hline 1341.3 & 1319.9 & 1348.9 & 1366.0 & 1403.7 & 1430.2 & 1421.1 & 1419.7 & 1533.6 \\
\hline
\end{tabular}




\begin{tabular}{|l|l|l|l|l|l|l|l|l|}
1404.1 & 1380.9 & 1406.4 & 1382.9 & 1441.0 & 1444.7 & 1430.0 & 1432.2 & 1534.3 \\
\hline 1417.0 & 1419.0 & 1417.3 & 1419.7 & 1444.7 & 1457.7 & 1449.7 & 1442.8 & 1540.1 \\
\hline 1441.8 & 1437.7 & 1426.1 & 1436.3 & 1465.7 & 1513.7 & 1462.9 & 1464.7 & 1768.0 \\
\hline 1451.1 & 1455.8 & 1456.1 & 1453.1 & 1528.7 & 1517.7 & 1529.8 & 1531.7 & 3052.1 \\
\hline 1505.5 & 1503.3 & 1504.5 & 1505.6 & 1533.7 & 1532.5 & 1532.8 & 1534.5 & 3056.1 \\
\hline 1538.7 & 1538.1 & 1538.6 & 1534.4 & 1543.7 & 1533.4 & 1545.2 & 1546.2 & 3066.7 \\
\hline 1540.9 & 1548.0 & 1544.7 & 1543.5 & 1548.1 & 1537.8 & 1553.7 & 1555.3 & 3157.1 \\
\hline 1551.2 & 1552.8 & 1551.1 & 1553.0 & 1555.5 & 1770.9 & 1779.2 & 1780.0 & 3165.3 \\
\hline 1769.5 & 1773.6 & 1771.6 & 1773.3 & 1848.6 & 2830.1 & 2900.2 & 2919.8 & 3167.6 \\
\hline 3113.5 & 3049.1 & 3114.7 & 3114.9 & 3109.6 & 3059.5 & 3109.8 & 3042.2 & 3174.6 \\
\hline 3118.7 & 3121.2 & 3122.1 & 3126.0 & 3110.8 & 3063.9 & 3111.8 & 3113.1 & 3209.1 \\
\hline 3127.1 & 3135.9 & 3131.3 & 3134.7 & 3124.5 & 3075.8 & 3115.2 & 3118.6 & 3225.5 \\
\hline 3174.9 & 3171.0 & 3173.5 & 3171.7 & 3134.7 & 3163.1 & 3195.5 & 3197.4 & \\
\hline 3180.5 & 3181.0 & 3176.2 & 3177.4 & 3200.6 & 3170.7 & 3204.1 & 3206.9 & \\
\hline 3196.2 & 3205.2 & 3199.5 & 3206.0 & 3202.6 & 3174.1 & 3207.5 & 3210.9 & \\
\hline 3209.0 & 3214.0 & 3211.4 & 3211.3 & 3210.1 & 3174.7 & 3218.0 & 3215.6 & \\
\hline 3221.1 & 3232.6 & 3236.2 & 3228.0 & 3215.8 & 3213.5 & 3221.1 & 3227.3 & \\
\hline 3280.2 & 3286.7 & 3282.3 & 3284.1 & 3225.4 & 3226.1 & 3268.1 & 3270.5 & \\
\hline
\end{tabular}

\begin{tabular}{|c|c|c|c|c|c|c|c|c|}
\hline P2 & P3 & P4 & P5 & P6 & P7 & P8 & P9 & P10 \\
\hline 48.0 & 51.8 & 46.9 & 46.9 & 46.9 & 43.8 & 43.8 & 43.3 & 37.6 \\
\hline 90.8 & 92.6 & 116.6 & 116.6 & 116.6 & 113.8 & 113.8 & 84.5 & 111.7 \\
\hline 156.5 & 156.8 & 152.6 & 152.5 & 152.6 & 144.3 & 144.4 & 111.1 & 149.3 \\
\hline 221.8 & 221.9 & 162.7 & 162.5 & 162.7 & 160.9 & 160.9 & 159.2 & 220.6 \\
\hline 259.1 & 259.4 & 238.7 & 238.7 & 238.7 & 245.2 & 245.1 & 247.1 & 252.7 \\
\hline 279.5 & 267.9 & 270.9 & 271.0 & 271.0 & 268.6 & 268.6 & 267.6 & 264.3 \\
\hline 319.8 & 326.1 & 340.4 & 340.3 & 340.3 & 340.1 & 340.1 & 327.4 & 361.2 \\
\hline 361.2 & 367.7 & 365.1 & 365.0 & 365.0 & 370.9 & 370.9 & 339.9 & 368.8 \\
\hline 413.7 & 430.1 & 414.1 & 414.1 & 414.2 & 415.5 & 415.7 & 430.8 & 432.8 \\
\hline 467.1 & 462.9 & 455.8 & 455.8 & 455.8 & 446.9 & 446.9 & 450.8 & 454.7 \\
\hline 648.1 & 631.0 & 546.3 & 546.2 & 546.6 & 528.5 & 528.9 & 529.8 & 749.5 \\
\hline 765.6 & 767.0 & 769.4 & 769.4 & 769.4 & 771.6 & 771.6 & 766.7 & 874.3 \\
\hline 885.9 & 881.1 & 896.0 & 896.0 & 896.0 & 891.1 & 891.0 & 879.6 & 923.9 \\
\hline 952.1 & 952.1 & 926.0 & 926.1 & 926.1 & 932.4 & 932.4 & 925.8 & 948.9 \\
\hline 966.3 & 984.6 & 967.6 & 967.6 & 967.6 & 960.0 & 960.0 & 948.5 & 960.2 \\
\hline 998.9 & 1010.0 & 996.1 & 996.1 & 996.1 & 987.7 & 987.6 & 985.3 & 1003.7 \\
\hline 1021.9 & 1021.4 & 1016.7 & 1016.7 & 1016.8 & 1019.7 & 1019.7 & 1019.0 & 1012.3 \\
\hline
\end{tabular}




\begin{tabular}{|l|l|l|l|l|l|l|l|l|}
\hline 1074.0 & 1074.6 & 1040.9 & 1040.9 & 1040.9 & 1040.8 & 1040.8 & 1040.6 & 1174.6 \\
\hline 1147.4 & 1139.9 & 1112.4 & 1112.5 & 1112.5 & 1107.3 & 1107.3 & 1143.8 & 1184.0 \\
\hline 1203.6 & 1191.1 & 1191.1 & 1191.2 & 1191.2 & 1205.2 & 1205.1 & 1188.0 & 1204.0 \\
\hline 1215.0 & 1212.7 & 1224.8 & 1224.8 & 1224.8 & 1223.6 & 1223.6 & 1206.7 & 1236.2 \\
\hline 1236.4 & 1242.7 & 1231.6 & 1231.7 & 1231.7 & 1231.5 & 1231.4 & 1230.1 & 1286.5 \\
\hline 1329.9 & 1340.3 & 1276.8 & 1276.8 & 1276.8 & 1288.2 & 1288.2 & 1294.9 & 1352.1 \\
\hline 1365.0 & 1372.5 & 1338.6 & 1338.7 & 1338.6 & 1320.3 & 1320.3 & 1353.3 & 1402.6 \\
\hline 1424.7 & 1423.7 & 1387.0 & 1387.0 & 1387.0 & 1389.4 & 1389.3 & 1376.8 & 1439.5 \\
\hline 1430.1 & 1433.0 & 1419.9 & 1420.0 & 1420.0 & 1419.9 & 1419.9 & 1420.7 & 1443.5 \\
\hline 1449.2 & 1442.0 & 1433.6 & 1433.6 & 1433.6 & 1430.6 & 1430.6 & 1431.8 & 1463.8 \\
\hline 1460.8 & 1461.8 & 1450.5 & 1450.5 & 1450.5 & 1450.7 & 1450.7 & 1446.9 & 1529.0 \\
\hline 1530.9 & 1532.1 & 1515.6 & 1515.6 & 1515.7 & 1519.1 & 1519.2 & 1520.0 & 1533.5 \\
\hline 1532.6 & 1533.7 & 1541.0 & 1541.0 & 1541.0 & 1539.4 & 1539.4 & 1533.8 & 1544.3 \\
\hline 1544.9 & 1544.7 & 1544.2 & 1544.2 & 1544.2 & 1546.5 & 1546.5 & 1543.4 & 1548.3 \\
\hline 1554.5 & 1556.6 & 1549.1 & 1549.1 & 1549.1 & 1549.5 & 1549.5 & 1549.3 & 1555.3 \\
\hline 1774.8 & 1774.8 & 1770.5 & 1770.5 & 1770.5 & 1770.3 & 1770.3 & 1770.1 & 1827.0 \\
\hline 3108.1 & 3042.6 & 3047.9 & 3047.7 & 3047.8 & 3046.4 & 3046.5 & 3113.6 & 3108.2 \\
\hline 3110.8 & 3112.7 & 3113.6 & 3113.6 & 3113.6 & 3114.9 & 3114.9 & 3126.4 & 3109.7 \\
\hline 3113.8 & 3114.6 & 3132.8 & 3132.8 & 3132.8 & 3133.9 & 3133.8 & 3134.3 & 3121.2 \\
\hline 3183.7 & 3184.2 & 3166.9 & 3166.9 & 3166.9 & 3166.5 & 3166.5 & 3166.2 & 3130.0 \\
\hline 3201.1 & 3206.3 & 3201.4 & 3201.5 & 3201.5 & 3202.5 & 3202.5 & 3204.7 & 3198.4 \\
\hline 3205.5 & 3208.1 & 3209.1 & 3209.2 & 3209.1 & 3206.5 & 3206.5 & 3210.0 & 3199.1 \\
\hline 3217.6 & 3213.7 & 3215.1 & 3215.1 & 3215.1 & 3226.8 & 3226.8 & 3224.6 & 3204.9 \\
\hline 3218.3 & 3223.8 & 3250.4 & 3250.5 & 3250.5 & 3245.7 & 3245.6 & 3244.1 & 3212.8 \\
\hline 3281.7 & 3290.1 & 3369.9 & 3369.9 & 3369.9 & 3363.3 & 3363.2 & 3365.0 & 3224.6 \\
\hline
\end{tabular}

Table S4: Optimized geometries in Cartesian Coordinates of reactants, reactive complexes, transition states and products for the reaction of isobutyl formate with $\mathrm{Cl}$ atoms at $\mathrm{MP} 2 / 6-31+\mathrm{G}$ $(d, p)$ level of theory. 


\begin{tabular}{|c|c|c|c|c|c|}
\hline \multicolumn{7}{|c|}{ Isobutyl formate } \\
\hline Center & Atomic & Atomic & \multicolumn{2}{|c|}{ Coordinates (Angstroms) } \\
\cline { 4 - 6 } Number & Number & Type & $\mathrm{X}$ & $\mathrm{Y}$ & $\mathrm{Z}$ \\
\hline 1 & 6 & 0 & 0.051806 & -0.44619 & 0.019134 \\
\hline 2 & 6 & 0 & -1.34565 & 0.018186 & -0.33991 \\
\hline 3 & 1 & 0 & -1.36109 & 0.203974 & -1.41856 \\
\hline 4 & 1 & 0 & 0.311058 & -1.38023 & -0.4814 \\
\hline 5 & 1 & 0 & 0.162593 & -0.58935 & 1.096587 \\
\hline 6 & 6 & 0 & -1.70917 & 1.309037 & 0.387977 \\
\hline 7 & 6 & 0 & -2.34017 & -1.0978 & -0.02149 \\
\hline 8 & 1 & 0 & -2.71049 & 1.637477 & 0.106954 \\
\hline 9 & 1 & 0 & -1.69861 & 1.150446 & 1.469153 \\
\hline 10 & 1 & 0 & -1.00756 & 2.107796 & 0.152211 \\
\hline 11 & 1 & 0 & -2.33351 & -1.32643 & 1.046515 \\
\hline 12 & 1 & 0 & -3.35197 & -0.7947 & -0.29092 \\
\hline 13 & 1 & 0 & -2.10415 & -2.01272 & -0.56667 \\
\hline 14 & 8 & 0 & 0.985978 & 0.581405 & -0.41064 \\
\hline 15 & 6 & 0 & 2.281586 & 0.319804 & -0.1462 \\
\hline 16 & 8 & 0 & 2.709721 & -0.67648 & 0.410681 \\
\hline 17 & 1 & 0 & 2.897676 & 1.146123 & -0.51131 \\
\hline
\end{tabular}

\begin{tabular}{|c|c|c|c|c|c|}
\hline \multicolumn{7}{|c|}{ RC1 } \\
\hline Center & Atomic & Atomic & \multicolumn{2}{|c|}{ Coordinates (Angstroms) } \\
\cline { 4 - 6 } Number & Number & Type & $\mathrm{X}$ & $\mathrm{Y}$ & $\mathrm{Z}$ \\
\hline 1 & 6 & 0 & -0.64132 & -0.8553 & -0.29291 \\
\hline 2 & 6 & 0 & -1.55542 & 0.344093 & -0.14166 \\
\hline 3 & 1 & 0 & -1.09564 & 1.181801 & -0.6739 \\
\hline 4 & 1 & 0 & -0.49839 & -1.13206 & -1.33844 \\
\hline 5 & 1 & 0 & -1.02564 & -1.72269 & 0.248051 \\
\hline 6 & 6 & 0 & -1.73309 & 0.727872 & 1.32437 \\
\hline 7 & 6 & 0 & -2.89786 & 0.029312 & -0.80113 \\
\hline 8 & 1 & 0 & -2.38293 & 1.599079 & 1.413816 \\
\hline 9 & 1 & 0 & -2.1939 & -0.0939 & 1.878253 \\
\hline 10 & 1 & 0 & -0.77877 & 0.964028 & 1.792679 \\
\hline 11 & 1 & 0 & -3.38316 & -0.81414 & -0.30516 \\
\hline 12 & 1 & 0 & -3.56584 & 0.887632 & -0.7296 \\
\hline 13 & 1 & 0 & -2.77788 & -0.21892 & -1.85663 \\
\hline 14 & 8 & 0 & 0.657484 & -0.50701 & 0.268201 \\
\hline 15 & 6 & 0 & 1.564168 & -1.51211 & 0.283956 \\
\hline
\end{tabular}




\begin{tabular}{|c|c|c|c|c|c|}
\hline 16 & 8 & 0 & 1.371389 & -2.6446 & -0.11819 \\
\hline 17 & 1 & 0 & 2.498918 & -1.14741 & 0.717576 \\
\hline 18 & 17 & 0 & 1.797254 & 1.95919 & -0.26956 \\
\hline
\end{tabular}

\begin{tabular}{|c|c|c|c|c|c|}
\hline \multicolumn{6}{|c|}{ RC2 } \\
\hline \multirow{2}{*}{$\begin{array}{l}\text { Center } \\
\text { Number }\end{array}$} & \multirow{2}{*}{$\begin{array}{l}\text { Atomic } \\
\text { Number }\end{array}$} & \multirow{2}{*}{$\begin{array}{l}\text { Atomic } \\
\text { Type }\end{array}$} & \multicolumn{3}{|c|}{ Coordinates (Angstroms) } \\
\hline & & & $\mathrm{X}$ & $\mathrm{Y}$ & $\mathrm{Z}$ \\
\hline 1 & 6 & 0 & 0.641375 & -0.85532 & 0.29288 \\
\hline 2 & 6 & 0 & 1.555437 & 0.344121 & 0.141606 \\
\hline 3 & 1 & 0 & 1.095464 & 1.181876 & 0.673677 \\
\hline 4 & 1 & 0 & 0.498419 & -1.13203 & 1.338383 \\
\hline 5 & 1 & 0 & 1.025798 & -1.72263 & -0.24813 \\
\hline 6 & 6 & 0 & 1.733155 & 0.727787 & -1.32444 \\
\hline 7 & 6 & 0 & 2.897802 & 0.0296 & 0.801267 \\
\hline 8 & 1 & 0 & 2.382682 & 1.599227 & -1.41391 \\
\hline 9 & 1 & 0 & 2.194359 & -0.09388 & -1.87815 \\
\hline 10 & 1 & 0 & 0.778818 & 0.963506 & -1.79292 \\
\hline 11 & 1 & 0 & 3.383014 & -0.81418 & 0.305748 \\
\hline 12 & 1 & 0 & 3.565898 & 0.887795 & 0.729285 \\
\hline 13 & 1 & 0 & 2.777819 & -0.21805 & 1.856898 \\
\hline 14 & 8 & 0 & -0.65745 & -0.50712 & -0.26813 \\
\hline 15 & 6 & 0 & -1.56414 & -1.51226 & -0.28372 \\
\hline 16 & 8 & 0 & -1.37123 & -2.64484 & 0.11807 \\
\hline 17 & 1 & 0 & -2.49902 & -1.14746 & -0.71695 \\
\hline 18 & 17 & 0 & -1.79739 & 1.959291 & 0.269467 \\
\hline
\end{tabular}

\begin{tabular}{|l|l|l|l|}
\hline \multicolumn{3}{|c|}{ RC3 } \\
\hline Center & Atomic & Atomic & Coordinates (Angstroms) \\
\hline
\end{tabular}




\begin{tabular}{|c|c|c|c|c|c|}
\hline Number & Number & Type & $\mathrm{X}$ & $\mathrm{Y}$ & $\mathrm{Z}$ \\
\hline 1 & 6 & 0 & -0.59642 & -0.05429 & 0.200112 \\
\hline 2 & 6 & 0 & -2.05412 & 0.342227 & 0.318023 \\
\hline 3 & 1 & 0 & -2.43456 & -0.08314 & 1.252157 \\
\hline 4 & 1 & 0 & 0.002227 & 0.349593 & 1.017318 \\
\hline 5 & 1 & 0 & -0.16489 & 0.279618 & -0.74594 \\
\hline 6 & 6 & 0 & -2.87713 & -0.20425 & -0.84489 \\
\hline 7 & 6 & 0 & -2.14937 & 1.865521 & 0.399075 \\
\hline 8 & 1 & 0 & -3.92521 & 0.077186 & -0.73666 \\
\hline 9 & 1 & 0 & -2.51519 & 0.205729 & -1.79097 \\
\hline 10 & 1 & 0 & -2.81798 & -1.29044 & -0.89718 \\
\hline 11 & 1 & 0 & -1.75318 & 2.324858 & -0.50911 \\
\hline 12 & 1 & 0 & -3.18875 & 2.176615 & 0.504344 \\
\hline 13 & 1 & 0 & -1.59007 & 2.257308 & 1.249689 \\
\hline 14 & 8 & 0 & -0.52534 & -1.50783 & 0.253941 \\
\hline 15 & 6 & 0 & 0.709805 & -2.02591 & 0.154469 \\
\hline 16 & 8 & 0 & 1.746919 & -1.39484 & 0.02163 \\
\hline 17 & 1 & 0 & 0.65409 & -3.11615 & 0.210666 \\
\hline 18 & 17 & 0 & 2.927309 & 1.20591 & -0.18351 \\
\hline
\end{tabular}

\begin{tabular}{|c|c|c|c|c|c|}
\hline \multicolumn{7}{|c|}{ TS1 } \\
\hline Center & Atomic & Atomic & \multicolumn{2}{|c|}{ Coordinates (Angstroms) } \\
\cline { 4 - 6 } Number & Number & Type & $\mathrm{X}$ & $\mathrm{Y}$ & $\mathrm{Z}$ \\
\hline 1 & 6 & 0 & -0.66685 & 0.62532 & -0.41541 \\
\hline 2 & 6 & 0 & 0.761679 & 0.856359 & -0.00832 \\
\hline 3 & 1 & 0 & 1.248797 & -0.37081 & -0.01567 \\
\hline 4 & 1 & 0 & -0.73301 & 0.097543 & -1.36755 \\
\hline 5 & 1 & 0 & -1.19056 & 1.583187 & -0.51785 \\
\hline 6 & 6 & 0 & 0.986479 & 1.315493 & 1.408269 \\
\hline 7 & 6 & 0 & 1.574604 & 1.55842 & -1.06674 \\
\hline 8 & 1 & 0 & 2.051829 & 1.353236 & 1.632463 \\
\hline 9 & 1 & 0 & 0.577295 & 2.324991 & 1.528157 \\
\hline 10 & 1 & 0 & 0.494592 & 0.659887 & 2.123054 \\
\hline 11 & 1 & 0 & 1.257003 & 2.603717 & -1.14016 \\
\hline 12 & 1 & 0 & 2.632722 & 1.546477 & -0.80889 \\
\hline 13 & 1 & 0 & 1.448865 & 1.091965 & -2.0435 \\
\hline 14 & 8 & 0 & -1.30967 & -0.15764 & 0.613078 \\
\hline 15 & 6 & 0 & -2.57979 & -0.52463 & 0.328222 \\
\hline
\end{tabular}




\begin{tabular}{|c|c|c|c|c|c|}
\hline 16 & 8 & 0 & -3.19289 & -0.22303 & -0.6794 \\
\hline 17 & 1 & 0 & -2.96882 & -1.13362 & 1.147501 \\
\hline 18 & 17 & 0 & 1.80853 & -1.74688 & -0.08724 \\
\hline
\end{tabular}

\begin{tabular}{|c|c|c|c|c|c|}
\hline \multicolumn{6}{|c|}{ TS2 } \\
\hline \multirow{2}{*}{$\begin{array}{l}\text { Center } \\
\text { Number }\end{array}$} & \multirow{2}{*}{$\begin{array}{l}\text { Atomic } \\
\text { Number }\end{array}$} & \multirow{2}{*}{$\begin{array}{l}\text { Atomic } \\
\text { Type }\end{array}$} & \multicolumn{3}{|c|}{ Coordinates (Angstroms) } \\
\hline & & & $\mathrm{X}$ & $\mathrm{Y}$ & $\mathrm{Z}$ \\
\hline 1 & 6 & 0 & 0.140871 & -0.24214 & 0.182658 \\
\hline 2 & 6 & 0 & 1.55944 & -0.03514 & -0.2648 \\
\hline 3 & 1 & 0 & 1.529268 & 0.35501 & -1.28635 \\
\hline 4 & 1 & 0 & -0.43574 & 0.938818 & 0.087714 \\
\hline 5 & 1 & 0 & -0.03315 & -0.49413 & 1.229878 \\
\hline 6 & 6 & 0 & 2.300735 & -1.37853 & -0.27156 \\
\hline 7 & 6 & 0 & 2.250765 & 0.979396 & 0.644297 \\
\hline 8 & 1 & 0 & 3.326441 & -1.22747 & -0.6093 \\
\hline 9 & 1 & 0 & 2.334411 & -1.80314 & 0.733734 \\
\hline 10 & 1 & 0 & 1.8192 & -2.09306 & -0.93727 \\
\hline 11 & 1 & 0 & 2.26937 & 0.62044 & 1.675299 \\
\hline 12 & 1 & 0 & 3.281409 & 1.124085 & 0.32151 \\
\hline 13 & 1 & 0 & 1.746574 & 1.944398 & 0.622162 \\
\hline 14 & 8 & 0 & -0.57848 & -1.049 & -0.70259 \\
\hline 15 & 6 & 0 & -1.71084 & -1.65008 & -0.20518 \\
\hline 16 & 8 & 0 & -2.10012 & -1.57409 & 0.939043 \\
\hline 17 & 1 & 0 & -2.18649 & -2.20874 & -1.01269 \\
\hline 18 & 17 & 0 & -1.1452 & 2.222789 & -0.18993 \\
\hline
\end{tabular}

\begin{tabular}{|l|l|l|l|}
\hline \multicolumn{3}{|c|}{ TS3 } \\
\hline Center & Atomic & Atomic & Coordinates (Angstroms) \\
\hline
\end{tabular}




\begin{tabular}{|c|c|c|c|c|c|}
\hline Number & Number & Type & $\mathrm{X}$ & $\mathrm{Y}$ & $\mathrm{Z}$ \\
\hline 1 & 6 & 0 & -0.08653 & -0.47291 & -0.35634 \\
\hline 2 & 6 & 0 & 1.284213 & -1.05637 & -0.15658 \\
\hline 3 & 1 & 0 & 1.160048 & -2.13675 & -0.32004 \\
\hline 4 & 1 & 0 & -0.50412 & -0.4899 & -1.36294 \\
\hline 5 & 1 & 0 & 0.038788 & 0.836177 & -0.16036 \\
\hline 6 & 6 & 0 & 1.816296 & -0.84135 & 1.258736 \\
\hline 7 & 6 & 0 & 2.239394 & -0.51255 & -1.21833 \\
\hline 8 & 1 & 0 & 2.778547 & -1.34184 & 1.367332 \\
\hline 9 & 1 & 0 & 1.959499 & 0.221486 & 1.455794 \\
\hline 10 & 1 & 0 & 1.133681 & -1.24269 & 2.005859 \\
\hline 11 & 1 & 0 & 2.405851 & 0.555687 & -1.07677 \\
\hline 12 & 1 & 0 & 3.201653 & -1.01824 & -1.14371 \\
\hline 13 & 1 & 0 & 1.850244 & -0.67106 & -2.22488 \\
\hline 14 & 8 & 0 & -0.99504 & -0.89168 & 0.617949 \\
\hline 15 & 6 & 0 & -2.32927 & -0.79303 & 0.295825 \\
\hline 16 & 8 & 0 & -2.76834 & -0.41759 & -0.76825 \\
\hline 17 & 1 & 0 & -2.91486 & -1.11832 & 1.157 \\
\hline 18 & 17 & 0 & 0.085469 & 2.290404 & 0.150894 \\
\hline
\end{tabular}

\begin{tabular}{|c|c|c|c|c|c|}
\hline \multicolumn{7}{|c|}{ TS4 } \\
\hline Center & Atomic & Atomic & \multicolumn{2}{|c|}{ Coordinates (Angstroms) } \\
\cline { 4 - 6 } Number & Number & Type & $\mathrm{X}$ & $\mathrm{Y}$ & $\mathrm{Z}$ \\
\hline 1 & 6 & 0 & -1.27192 & 0.585554 & 0.008688 \\
\hline 2 & 6 & 0 & 0.23896 & 0.552824 & -0.19317 \\
\hline 3 & 1 & 0 & 0.41969 & 0.195604 & -1.21139 \\
\hline 4 & 1 & 0 & -1.74133 & 1.279242 & -0.69014 \\
\hline 5 & 1 & 0 & -1.53924 & 0.882222 & 1.025006 \\
\hline 6 & 6 & 0 & 0.866077 & -0.41253 & 0.774751 \\
\hline 7 & 6 & 0 & 0.804771 & 1.964717 & -0.04488 \\
\hline 8 & 1 & 0 & 2.182894 & -0.51713 & 0.352087 \\
\hline 9 & 1 & 0 & 1.00829 & -0.05597 & 1.792964 \\
\hline 10 & 1 & 0 & 0.562707 & -1.45194 & 0.700544 \\
\hline 11 & 1 & 0 & 0.614254 & 2.352593 & 0.957741 \\
\hline 12 & 1 & 0 & 1.880571 & 1.964092 & -0.21121 \\
\hline 13 & 1 & 0 & 0.348158 & 2.642781 & -0.76595 \\
\hline 14 & 8 & 0 & -1.77539 & -0.75103 & -0.2415 \\
\hline 15 & 6 & 0 & -3.1196 & -0.87327 & -0.16112 \\
\hline
\end{tabular}




\begin{tabular}{|c|c|c|c|c|c|}
\hline 16 & 8 & 0 & -3.89683 & 0.024933 & 0.106564 \\
\hline 17 & 1 & 0 & -3.4002 & -1.9077 & -0.37477 \\
\hline 18 & 17 & 0 & 3.525426 & -0.6164 & -0.16477 \\
\hline
\end{tabular}

\begin{tabular}{|c|c|c|c|c|c|}
\hline \multicolumn{7}{|c|}{ TS5 } \\
\hline Center & Atomic & Atomic & \multicolumn{2}{|c|}{ Coordinates (Angstroms) } \\
\cline { 4 - 6 } Number & Number & Type & $\mathrm{X}$ & $\mathrm{Y}$ & $\mathrm{Z}$ \\
\hline 1 & 6 & 0 & 0.787752 & 0.551926 & -0.36703 \\
\hline 2 & 6 & 0 & -0.33002 & 1.375031 & 0.248691 \\
\hline 3 & 1 & 0 & 0.146093 & 2.188689 & 0.816363 \\
\hline 4 & 1 & 0 & 1.403699 & 1.161962 & -1.02809 \\
\hline 5 & 1 & 0 & 0.402027 & -0.29684 & -0.93375 \\
\hline 6 & 6 & 0 & -1.13376 & 0.593049 & 1.249071 \\
\hline 7 & 6 & 0 & -1.19517 & 1.995177 & -0.84914 \\
\hline 8 & 1 & 0 & -2.02978 & 1.077171 & 1.628117 \\
\hline 9 & 1 & 0 & -1.71113 & -0.45845 & 0.53217 \\
\hline 10 & 1 & 0 & -0.57804 & 0.028423 & 1.991026 \\
\hline 11 & 1 & 0 & -1.65556 & 1.222567 & -1.46514 \\
\hline 12 & 1 & 0 & -1.98987 & 2.599569 & -0.41227 \\
\hline 13 & 1 & 0 & -0.59574 & 2.638136 & -1.49357 \\
\hline 14 & 8 & 0 & 1.609613 & 0.057466 & 0.722119 \\
\hline 15 & 6 & 0 & 2.707628 & -0.63097 & 0.335171 \\
\hline 16 & 8 & 0 & 3.041866 & -0.84227 & -0.81595 \\
\hline 17 & 1 & 0 & 3.251949 & -0.96645 & 1.221679 \\
\hline 18 & 17 & 0 & -2.28671 & -1.54245 & -0.22391 \\
\hline
\end{tabular}




\begin{tabular}{|c|c|c|c|c|c|}
\hline \multicolumn{6}{|c|}{ TS6 } \\
\hline \multirow{2}{*}{$\begin{array}{l}\text { Center } \\
\text { Number }\end{array}$} & \multirow{2}{*}{$\begin{array}{l}\text { Atomic } \\
\text { Number }\end{array}$} & \multirow{2}{*}{$\begin{array}{l}\text { Atomic } \\
\text { Type }\end{array}$} & \multicolumn{3}{|c|}{ Coordinates (Angstroms) } \\
\hline & & & $\mathrm{X}$ & $\mathrm{Y}$ & Z \\
\hline 1 & 6 & 0 & 1.103172 & -0.70798 & -0.05897 \\
\hline 2 & 6 & 0 & -0.2296 & -1.39721 & -0.27598 \\
\hline 3 & 1 & 0 & -0.58862 & -1.14193 & -1.27808 \\
\hline 4 & 1 & 0 & 1.822989 & -0.9729 & -0.83415 \\
\hline 5 & 1 & 0 & 1.531689 & -0.96714 & 0.912642 \\
\hline 6 & 6 & 0 & -1.25379 & -0.95697 & 0.735125 \\
\hline 7 & 6 & 0 & -0.0388 & -2.92027 & -0.20705 \\
\hline 8 & 1 & 0 & -2.19018 & -1.50849 & 0.729308 \\
\hline 9 & 1 & 0 & -0.89053 & -0.72017 & 1.732919 \\
\hline 10 & 1 & 0 & -1.72115 & 0.269127 & 0.329056 \\
\hline 11 & 1 & 0 & 0.295004 & -3.22617 & 0.785602 \\
\hline 12 & 1 & 0 & -0.97341 & -3.4344 & -0.42718 \\
\hline 13 & 1 & 0 & 0.707321 & -3.24326 & -0.93422 \\
\hline 14 & 8 & 0 & 0.857362 & 0.716265 & -0.0993 \\
\hline 15 & 6 & 0 & 1.95921 & \begin{tabular}{|l}
1.489923 \\
\end{tabular} & 0.006816 \\
\hline 16 & 8 & 0 & 3.099902 & 1.081417 & 0.135835 \\
\hline 17 & 1 & 0 & 1.665245 & 2.541029 & -0.0415 \\
\hline 18 & 17 & 0 & -2.38575 & 1.469287 & -0.14508 \\
\hline
\end{tabular}

\begin{tabular}{|c|c|c|c|c|c|}
\hline \multicolumn{7}{|c|}{ TS7 } \\
\hline \multirow{2}{*}{$\begin{array}{c}\text { Center } \\
\text { Number }\end{array}$} & Atomic & Atomic & \multicolumn{2}{|c|}{ Coordinates (Angstroms) } \\
\cline { 4 - 6 } & & Type & $\mathrm{X}$ & $\mathrm{Y}$ & $\mathrm{Z}$ \\
\hline 1 & 6 & 0 & 1.103172 & -0.70798 & -0.05897 \\
\hline 2 & 6 & 0 & -0.2296 & -1.39721 & -0.27598 \\
\hline 3 & 1 & 0 & -0.58862 & -1.14193 & -1.27808 \\
\hline 4 & 1 & 0 & 1.822989 & -0.9729 & -0.83415 \\
\hline 5 & 1 & 0 & 1.531689 & -0.96714 & 0.912642 \\
\hline 6 & 6 & 0 & -1.25379 & -0.95697 & 0.735125 \\
\hline 7 & 6 & 0 & -0.0388 & -2.92027 & -0.20705 \\
\hline 8 & 1 & 0 & -2.19018 & -1.50849 & 0.729308 \\
\hline 9 & 1 & 0 & -0.89053 & -0.72017 & 1.732919 \\
\hline 10 & 1 & 0 & -1.72115 & 0.269127 & 0.329056 \\
\hline 11 & 1 & 0 & 0.295004 & -3.22617 & 0.785602 \\
\hline
\end{tabular}




\begin{tabular}{|c|c|c|c|c|c|}
\hline 12 & 1 & 0 & -0.97341 & -3.4344 & -0.42718 \\
\hline 13 & 1 & 0 & 0.707321 & -3.24326 & -0.93422 \\
\hline 14 & 8 & 0 & 0.857362 & 0.716265 & -0.0993 \\
\hline 15 & 6 & 0 & 1.95921 & 1.489923 & 0.006816 \\
\hline 16 & 8 & 0 & 3.099902 & 1.081417 & 0.135835 \\
\hline 17 & 1 & 0 & 1.665245 & 2.541029 & -0.0415 \\
\hline 18 & 17 & 0 & -2.38575 & 1.469287 & -0.14508 \\
\hline
\end{tabular}

\begin{tabular}{|c|c|c|c|c|c|}
\hline \multicolumn{7}{|c|}{ TS8 } \\
\hline Center & Atomic & Atomic & \multicolumn{2}{|c|}{ Coordinates (Angstroms) } \\
\cline { 4 - 6 } Number & Number & Type & $X$ & Y & $Z$ \\
\hline 1 & 6 & 0 & 1.178928 & -0.4322 & 0.161472 \\
\hline 2 & 6 & 0 & -0.24705 & 0.087956 & 0.004995 \\
\hline 3 & 1 & 0 & -0.43426 & 0.183379 & -1.06939 \\
\hline 4 & 1 & 0 & 1.306836 & -1.41474 & -0.29455 \\
\hline 5 & 1 & 0 & 1.47221 & -0.49342 & 1.211372 \\
\hline 6 & 6 & 0 & -0.42952 & 1.446965 & 0.67425 \\
\hline 7 & 6 & 0 & -1.18789 & -0.94618 & 0.564265 \\
\hline 8 & 1 & 0 & -1.44671 & 1.809765 & 0.533244 \\
\hline 9 & 1 & 0 & -0.23774 & 1.369919 & 1.746905 \\
\hline 10 & 1 & 0 & 0.259077 & 2.177009 & 0.253477 \\
\hline 11 & 1 & 0 & -1.32769 & -0.94925 & 1.642896 \\
\hline 12 & 1 & 0 & -2.43923 & -0.5373 & 0.147583 \\
\hline 13 & 1 & 0 & -1.15573 & -1.93677 & 0.118123 \\
\hline 14 & 8 & 0 & 2.047463 & 0.510879 & -0.51566 \\
\hline 15 & 6 & 0 & 3.365406 & 0.221813 & -0.43406 \\
\hline 16 & 8 & 0 & 3.846739 & -0.73326 & 0.148321 \\
\hline 17 & 1 & 0 & 3.933828 & 0.982674 & -0.97488 \\
\hline 18 & 17 & 0 & -3.7155 & -0.09896 & -0.3648 \\
\hline & & & & & \\
\hline
\end{tabular}




\begin{tabular}{|c|c|c|c|c|c|}
\hline \multicolumn{6}{|c|}{ TS9 } \\
\hline \multirow{2}{*}{$\begin{array}{l}\text { Center } \\
\text { Number }\end{array}$} & \multirow{2}{*}{$\begin{array}{l}\text { Atomic } \\
\text { Number }\end{array}$} & \multirow{2}{*}{$\begin{array}{c}\text { Atomic } \\
\text { Type }\end{array}$} & \multicolumn{3}{|c|}{ Coordinates (Angstroms) } \\
\hline & & & $\mathrm{X}$ & $\mathrm{Y}$ & Z \\
\hline 1 & 6 & 0 & 0.639473 & -0.00582 & 0.25158 \\
\hline 2 & 6 & 0 & -0.2731 & 1.120961 & -0.19836 \\
\hline 3 & 1 & 0 & -0.53576 & 0.947636 & -1.24628 \\
\hline 4 & 1 & 0 & 0.140235 & -0.97393 & 0.208871 \\
\hline 5 & 1 & 0 & 1.003275 & 0.158345 & 1.268256 \\
\hline 6 & 6 & 0 & 0.420326 & 2.487085 & -0.08941 \\
\hline 7 & 6 & 0 & -1.53201 & 1.124177 & 0.623815 \\
\hline 8 & 1 & 0 & -0.23327 & 3.281073 & -0.44965 \\
\hline 9 & 1 & 0 & 0.684464 & 2.701928 & 0.947678 \\
\hline 10 & 1 & 0 & 1.330835 & 2.489651 & -0.68675 \\
\hline 11 & 1 & 0 & -1.42143 & 0.982741 & 1.697008 \\
\hline 12 & 1 & 0 & -2.27892 & 1.865047 & 0.353542 \\
\hline 13 & 1 & 0 & -2.20258 & -0.03801 & 0.265319 \\
\hline 14 & 8 & 0 & 1.769046 & -0.0245 & -0.65682 \\
\hline 15 & 6 & 0 & 2.765964 & -0.86606 & -0.30428 \\
\hline 16 & 8 & 0 & 2.784472 & -1.56719 & 0.691042 \\
\hline 17 & 1 & 0 & 3.559061 & -0.8166 & -1.05477 \\
\hline 18 & 17 & 0 & -2.8587 & -1.23685 & -0.19277 \\
\hline
\end{tabular}

\begin{tabular}{|c|c|c|c|c|c|}
\hline \multicolumn{7}{|c|}{ TS10 } \\
\hline Center & Atomic & \multirow{2}{*}{$\begin{array}{c}\text { Atomic } \\
\text { Number }\end{array}$} & Number & Type & Coordinates (Angstroms) \\
\cline { 4 - 6 } & & $\mathrm{X}$ & $\mathrm{Y}$ & $\mathrm{Z}$ \\
\hline 1 & 6 & 0 & 1.181954 & 0.683034 & -0.0524 \\
\hline 2 & 6 & 0 & 2.195806 & -0.40193 & -0.34292 \\
\hline 3 & 1 & 0 & 1.960482 & -0.81224 & -1.3296 \\
\hline 4 & 1 & 0 & 1.216599 & 1.492683 & -0.78179 \\
\hline 5 & 1 & 0 & 1.297253 & 1.094697 & 0.952171 \\
\hline 6 & 6 & 0 & 2.126038 & -1.5217 & 0.691278 \\
\hline 7 & 6 & 0 & 3.58785 & 0.229122 & -0.39301 \\
\hline 8 & 1 & 0 & 2.854115 & -2.29858 & 0.457291 \\
\hline 9 & 1 & 0 & 2.355975 & -1.13465 & 1.686831 \\
\hline 10 & 1 & 0 & 1.137627 & -1.97786 & 0.718861 \\
\hline 11 & 1 & 0 & 3.846753 & 0.671214 & 0.571344 \\
\hline 12 & 1 & 0 & 4.335735 & -0.52916 & -0.6231 \\
\hline 13 & 1 & 0 & 3.649991 & 1.007601 & -1.15447 \\
\hline
\end{tabular}




\begin{tabular}{|l|c|c|c|c|c|}
\hline 14 & 8 & 0 & -0.15404 & 0.067355 & -0.13445 \\
\hline 15 & 6 & 0 & -1.15956 & 0.883492 & 0.128153 \\
\hline 16 & 8 & 0 & -1.18218 & 2.050318 & 0.428126 \\
\hline 17 & 1 & 0 & -2.1984 & 0.207549 & 0.012155 \\
\hline 18 & 17 & 0 & -3.37406 & -0.81734 & -0.17916 \\
\hline
\end{tabular}

\begin{tabular}{|c|c|c|c|c|c|}
\hline \multicolumn{6}{|c|}{ PC1 } \\
\hline Center & Atomic & Atomic & \multicolumn{2}{|c|}{ Coordinates (Angstroms) } \\
\cline { 4 - 6 } Number & Number & Type & $\mathrm{X}$ & $\mathrm{Y}$ & $\mathrm{Z}$ \\
\hline 1 & 6 & 0 & 0.791698 & -0.72805 & -0.41341 \\
\hline 2 & 6 & 0 & -0.56852 & -1.18252 & -0.00612 \\
\hline 3 & 1 & 0 & -1.36772 & 0.747851 & 0.037371 \\
\hline 4 & 1 & 0 & 0.805406 & -0.35013 & -1.43697 \\
\hline 5 & 1 & 0 & 1.535243 & -1.53216 & -0.34232 \\
\hline 6 & 6 & 0 & -0.78893 & -1.55967 & 1.423193 \\
\hline 7 & 6 & 0 & -1.40558 & -1.8362 & -1.05775 \\
\hline 8 & 1 & 0 & -1.85359 & -1.60272 & 1.654027 \\
\hline 9 & 1 & 0 & -0.37102 & -2.55568 & 1.624707 \\
\hline 10 & 1 & 0 & -0.31 & -0.85712 & 2.10336 \\
\hline 11 & 1 & 0 & -1.08155 & -2.87056 & -1.23381 \\
\hline 12 & 1 & 0 & -2.45131 & -1.87603 & -0.75192 \\
\hline 13 & 1 & 0 & -1.3425 & -1.30684 & -2.00908 \\
\hline 14 & 8 & 0 & 1.191526 & 0.340226 & 0.488377 \\
\hline 15 & 6 & 0 & 2.440257 & 0.814599 & 0.284653 \\
\hline 16 & 8 & 0 & 3.22077 & 0.415441 & -0.56084 \\
\hline 17 & 1 & 0 & 2.640704 & 1.615961 & 1.000197 \\
\hline 18 & 17 & 0 & -2.01856 & 1.852535 & -0.08525 \\
\hline & & & & & \\
\hline
\end{tabular}

\section{PC2}




\begin{tabular}{|c|c|c|c|c|c|}
\hline \multirow{2}{*}{$\begin{array}{c}\text { Center } \\
\text { Number }\end{array}$} & Atomic & Atomic & \multicolumn{3}{|c|}{ Coordinates (Angstroms) } \\
\cline { 4 - 6 } & Number & Type & $\mathrm{X}$ & $\mathrm{Y}$ & $\mathrm{Z}$ \\
\hline 1 & 6 & 0 & 0.441808 & -0.33105 & 0.32868 \\
\hline 2 & 6 & 0 & 1.485385 & 0.595077 & -0.19033 \\
\hline 3 & 1 & 0 & 1.063479 & 1.122328 & -1.05253 \\
\hline 4 & 1 & 0 & -1.28333 & 0.97502 & 0.244478 \\
\hline 5 & 1 & 0 & 0.475891 & -0.75977 & 1.323232 \\
\hline 6 & 6 & 0 & 2.721561 & -0.18268 & -0.67017 \\
\hline 7 & 6 & 0 & 1.856808 & 1.615726 & 0.885199 \\
\hline 8 & 1 & 0 & 3.457705 & 0.507158 & -1.08482 \\
\hline 9 & 1 & 0 & 3.183619 & -0.71542 & 0.163003 \\
\hline 10 & 1 & 0 & 2.454057 & -0.9057 & -1.4397 \\
\hline 11 & 1 & 0 & 2.252499 & 1.113672 & 1.770209 \\
\hline 12 & 1 & 0 & 2.625703 & 2.291546 & 0.511423 \\
\hline 13 & 1 & 0 & 0.996221 & 2.213506 & 1.185006 \\
\hline 14 & 8 & 0 & -0.12381 & -1.13882 & -0.65144 \\
\hline 15 & 6 & 0 & -0.88435 & -2.19804 & -0.23097 \\
\hline 16 & 8 & 0 & -1.08211 & -2.50737 & 0.925206 \\
\hline 17 & 1 & 0 & -1.27806 & -2.71192 & -1.10932 \\
\hline 18 & 17 & 0 & -2.23692 & 1.708524 & -0.20209 \\
\hline
\end{tabular}

\begin{tabular}{|c|c|c|c|c|c|}
\hline \multicolumn{7}{|c|}{ PC3 } \\
\hline Center & Atomic & Atomic & \multicolumn{2}{|c|}{ Coordinates (Angstroms) } \\
\cline { 4 - 6 } Number & Number & Type & $\mathrm{X}$ & $\mathrm{Y}$ & $\mathrm{Z}$ \\
\hline 1 & 6 & 0 & -0.43083 & -0.69333 & 0.31556 \\
\hline 2 & 6 & 0 & -1.84249 & -0.41702 & -0.07117 \\
\hline 3 & 1 & 0 & -2.2831 & -1.37111 & -0.39877 \\
\hline 4 & 1 & 0 & -0.15984 & -1.11105 & 1.277494 \\
\hline 5 & 1 & 0 & 0.732195 & 1.189277 & 0.424652 \\
\hline 6 & 6 & 0 & -1.94421 & 0.568103 & -1.23814 \\
\hline 7 & 6 & 0 & -2.61972 & 0.07561 & 1.149572 \\
\hline 8 & 1 & 0 & -2.98679 & 0.679491 & -1.53715 \\
\hline 9 & 1 & 0 & -1.56846 & 1.549841 & -0.94647 \\
\hline 10 & 1 & 0 & -1.37396 & 0.224518 & -2.09959 \\
\hline 11 & 1 & 0 & -2.2225 & 1.031206 & 1.495739 \\
\hline 12 & 1 & 0 & -3.66927 & 0.219276 & 0.894058 \\
\hline 13 & 1 & 0 & -2.56526 & -0.63763 & 1.97279 \\
\hline 14 & 8 & 0 & 0.387001 & -1.07133 & -0.74291 \\
\hline
\end{tabular}




\begin{tabular}{|c|c|c|c|c|c|}
\hline 15 & 6 & 0 & 1.594683 & -1.64019 & -0.42973 \\
\hline 16 & 8 & 0 & 1.993708 & -1.87048 & 0.692175 \\
\hline 17 & 1 & 0 & 2.129811 & -1.84865 & -1.35731 \\
\hline 18 & 17 & 0 & 1.551576 & 2.132372 & 0.136699 \\
\hline
\end{tabular}

\begin{tabular}{|c|c|c|c|c|c|}
\hline \multicolumn{7}{|c|}{ P1 } \\
\hline Center & Atomic & Atomic & \multicolumn{2}{|c|}{ Coordinates (Angstroms) } \\
\cline { 4 - 6 } Number & Number & Type & $\mathrm{X}$ & $\mathrm{Y}$ & $\mathrm{Z}$ \\
\hline 1 & 6 & 0 & 0.050397 & -0.45036 & 0.126728 \\
\hline 2 & 6 & 0 & -1.35232 & -0.02818 & -0.13625 \\
\hline 3 & 1 & 0 & 0.283212 & -1.40466 & -0.34754 \\
\hline 4 & 1 & 0 & 0.262392 & -0.55149 & 1.199498 \\
\hline 5 & 6 & 0 & -1.76324 & 1.350068 & 0.267039 \\
\hline 6 & 6 & 0 & -2.38596 & -1.10747 & -0.14931 \\
\hline 7 & 1 & 0 & -2.70555 & 1.627544 & -0.206 \\
\hline 8 & 1 & 0 & -1.91604 & 1.410849 & 1.354045 \\
\hline 9 & 1 & 0 & -1.00814 & 2.0873 & $-8.7 \mathrm{E}-05$ \\
\hline 10 & 1 & 0 & -2.65364 & -1.42221 & 0.868847 \\
\hline 11 & 1 & 0 & -3.30339 & -0.75888 & -0.62462 \\
\hline 12 & 1 & 0 & -2.03692 & -1.99316 & -0.68148 \\
\hline 13 & 8 & 0 & 0.947552 & 0.567568 & -0.40382 \\
\hline 14 & 6 & 0 & 2.257962 & 0.32297 & -0.20563 \\
\hline 15 & 8 & 0 & 2.726446 & -0.64956 & 0.361175 \\
\hline 16 & 1 & 0 & 2.845034 & 1.138403 & -0.637 \\
\hline
\end{tabular}

\begin{tabular}{|c|c|c|c|c|c|}
\hline \multicolumn{9}{|c|}{ P2 } \\
\hline Center & Atomic & \multirow{2}{*}{$\begin{array}{c}\text { Atomic } \\
\text { Number }\end{array}$} & Number & Type & Coordinates (Angstroms) \\
\cline { 4 - 6 } & & & $\mathrm{X}$ & $\mathrm{Y}$ & $\mathrm{Z}$ \\
\hline 1 & 6 & 0 & 0.037713 & -0.42922 & -0.10243 \\
\hline 2 & 6 & 0 & -1.35538 & 0.037879 & -0.33574 \\
\hline 3 & 1 & 0 & -1.43842 & 0.311785 & -1.39269 \\
\hline 4 & 1 & 0 & 0.329784 & -1.01092 & 0.761968 \\
\hline
\end{tabular}




\begin{tabular}{|c|c|c|c|c|c|}
\hline 5 & 6 & 0 & -1.67549 & 1.285335 & 0.50317 \\
\hline 6 & 6 & 0 & -2.33897 & -1.09358 & -0.03847 \\
\hline 7 & 1 & 0 & -2.68356 & 1.639784 & 0.28266 \\
\hline 8 & 1 & 0 & -1.62133 & 1.051334 & 1.56807 \\
\hline 9 & 1 & 0 & -0.97293 & 2.090188 & 0.289785 \\
\hline 10 & 1 & 0 & -2.26475 & -1.40112 & 1.006491 \\
\hline 11 & 1 & 0 & -3.36196 & -0.76284 & -0.21813 \\
\hline 12 & 1 & 0 & -2.14296 & -1.96284 & -0.66564 \\
\hline 13 & 8 & 0 & 1.00617 & 0.452257 & -0.58377 \\
\hline 14 & 6 & 0 & 2.293826 & 0.251714 & -0.18434 \\
\hline 15 & 8 & 0 & 2.676128 & -0.61062 & 0.581388 \\
\hline 16 & 1 & 0 & 2.927553 & 0.998728 & -0.66662 \\
\hline
\end{tabular}

\begin{tabular}{|c|c|c|c|c|c|}
\hline \multicolumn{7}{|c|}{$\mathbf{P 3}$} \\
\hline Center & \multirow{2}{*}{$\begin{array}{c}\text { Atomic } \\
\text { Number }\end{array}$} & $\begin{array}{c}\text { Atomic } \\
\text { Number }\end{array}$ & \multicolumn{2}{|c|}{ Coordinates (Angstroms) } \\
\cline { 4 - 6 } & & $\mathrm{X}$ & $\mathrm{Y}$ & $\mathrm{Z}$ \\
\hline 1 & 6 & 0 & 0.052992 & -0.35775 & 0.074231 \\
\hline 2 & 6 & 0 & -1.3385 & -0.02968 & -0.33983 \\
\hline 3 & 1 & 0 & -1.34867 & 0.089092 & -1.434 \\
\hline 4 & 1 & 0 & 0.454774 & -1.36148 & 0.069232 \\
\hline 5 & 6 & 0 & -1.81939 & 1.286434 & 0.276549 \\
\hline 6 & 6 & 0 & -2.26777 & -1.18722 & 0.024817 \\
\hline 7 & 1 & 0 & -2.82544 & 1.518429 & -0.07491 \\
\hline 8 & 1 & 0 & -1.84487 & 1.205646 & 1.364515 \\
\hline 9 & 1 & 0 & -1.16261 & 2.112638 & 0.008873 \\
\hline 10 & 1 & 0 & -2.29129 & -1.33011 & 1.106215 \\
\hline 11 & 1 & 0 & -3.28273 & -0.97668 & -0.31195 \\
\hline 12 & 1 & 0 & -1.94336 & -2.12027 & -0.43734 \\
\hline 13 & 8 & 0 & 0.975262 & 0.651887 & -0.19975 \\
\hline 14 & 6 & 0 & 2.297644 & 0.343845 & -0.07642 \\
\hline 15 & 8 & 0 & 2.750958 & -0.74028 & 0.231853 \\
\hline 16 & 1 & 0 & 2.884573 & 1.236084 & -0.30352 \\
\hline
\end{tabular}

\section{P4}




\begin{tabular}{|c|c|c|c|c|c|}
\hline Center & Atomic & Atomic & \multicolumn{3}{|c|}{ Coordinates (Angstroms) } \\
\cline { 4 - 6 } Number & Number & Type & $\mathrm{X}$ & $\mathrm{Y}$ & $\mathrm{Z}$ \\
\hline 1 & 6 & 0 & 0.016213 & -0.42221 & 0.038753 \\
\hline 2 & 6 & 0 & -1.378 & 0.070201 & -0.30939 \\
\hline 3 & 1 & 0 & -1.38498 & 0.25366 & -1.39422 \\
\hline 4 & 1 & 0 & 0.255258 & -1.3499 & -0.48281 \\
\hline 5 & 1 & 0 & 0.126875 & -0.58479 & 1.112279 \\
\hline 6 & 6 & 0 & -1.70795 & 1.339286 & 0.404297 \\
\hline 7 & 6 & 0 & -2.39711 & -1.0323 & -0.00962 \\
\hline 8 & 1 & 0 & -2.73997 & 1.612292 & 0.569317 \\
\hline 9 & 1 & 0 & -0.94946 & 2.089379 & 0.567384 \\
\hline 10 & 1 & 0 & -2.39214 & -1.28207 & 1.052419 \\
\hline 11 & 1 & 0 & -3.4016 & -0.70174 & -0.27276 \\
\hline 12 & 1 & 0 & -2.17741 & -1.93651 & -0.57833 \\
\hline 13 & 8 & 0 & 0.958819 & 0.601338 & -0.37858 \\
\hline 14 & 6 & 0 & 2.254558 & 0.312323 & -0.14025 \\
\hline 15 & 8 & 0 & 2.673564 & -0.7068 & 0.380329 \\
\hline 16 & 1 & 0 & 2.878095 & 1.139534 & -0.49003 \\
\hline
\end{tabular}

\begin{tabular}{|c|c|c|c|c|c|}
\hline \multicolumn{7}{|c|}{ P5 } \\
\hline Center & Atomic & Atomic & \multicolumn{2}{|c|}{ Coordinates (Angstroms) } \\
\cline { 4 - 6 } Number & Number & Type & $\mathrm{X}$ & $\mathrm{Y}$ & $\mathrm{Z}$ \\
\hline 1 & 6 & 0 & 0.016175 & -0.42255 & 0.037996 \\
\hline 2 & 6 & 0 & -1.37807 & 0.070333 & -0.30928 \\
\hline 3 & 1 & 0 & -1.38551 & 0.254228 & -1.39405 \\
\hline 4 & 1 & 0 & 0.254661 & -1.35016 & -0.48398 \\
\hline 5 & 1 & 0 & 0.127393 & -0.58546 & 1.1114 \\
\hline 6 & 6 & 0 & -1.70734 & 1.339373 & 0.404802 \\
\hline 7 & 6 & 0 & -2.39735 & -1.03193 & -0.00941 \\
\hline 8 & 1 & 0 & -2.73919 & 1.612633 & 0.570527 \\
\hline 9 & 1 & 0 & -0.94862 & 2.089244 & 0.567765 \\
\hline 10 & 1 & 0 & -2.39223 & -1.28174 & 1.052614 \\
\hline 11 & 1 & 0 & -3.40182 & -0.70116 & -0.27236 \\
\hline 12 & 1 & 0 & -2.17793 & -1.93617 & -0.5782 \\
\hline 13 & 8 & 0 & 0.958854 & 0.600812 & -0.3796 \\
\hline 14 & 6 & 0 & 2.254477 & 0.312223 & -0.14023 \\
\hline 15 & 8 & 0 & 2.673369 & -0.7065 & 0.381241 \\
\hline
\end{tabular}




\begin{tabular}{|l|l|l|l|l|l|}
\hline 16 & 1 & 0 & 2.878083 & 1.139343 & -0.4901 \\
\hline
\end{tabular}

\begin{tabular}{|c|c|c|c|c|c|}
\hline \multicolumn{7}{|c|}{ P6 } \\
\hline Center & Atomic & Atomic & \multicolumn{2}{|c|}{ Coordinates (Angstroms) } \\
\cline { 4 - 6 } Number & Number & Type & $\mathrm{X}$ & $\mathrm{Y}$ & $\mathrm{Z}$ \\
\hline 1 & 6 & 0 & 0.016149 & -0.42272 & 0.037659 \\
\hline 2 & 6 & 0 & -1.3781 & 0.07045 & -0.30928 \\
\hline 3 & 1 & 0 & -1.38576 & 0.254375 & -1.39403 \\
\hline 4 & 1 & 0 & 0.254399 & -1.35024 & -0.48459 \\
\hline 5 & 1 & 0 & 0.127591 & -0.58585 & 1.111002 \\
\hline 6 & 6 & 0 & -1.70694 & 1.339341 & 0.405146 \\
\hline 7 & 6 & 0 & -2.39748 & -1.03172 & -0.0093 \\
\hline 8 & 1 & 0 & -2.73872 & 1.612974 & 0.570701 \\
\hline 9 & 1 & 0 & -0.94807 & 2.089162 & 0.567643 \\
\hline 10 & 1 & 0 & -2.39187 & -1.28203 & 1.05261 \\
\hline 11 & 1 & 0 & -3.40202 & -0.70059 & -0.27154 \\
\hline 12 & 1 & 0 & -2.17861 & -1.93576 & -0.57862 \\
\hline 13 & 8 & 0 & 0.958833 & 0.600603 & -0.38 \\
\hline 14 & 6 & 0 & 2.254417 & 0.312168 & -0.1402 \\
\hline 15 & 8 & 0 & 2.673261 & -0.70641 & 0.381584 \\
\hline 16 & 1 & 0 & 2.87803 & 1.139289 & -0.49006 \\
\hline & & & & & \\
\hline
\end{tabular}

\begin{tabular}{|c|c|c|c|c|c|}
\hline \multicolumn{9}{|c|}{ P7 } \\
\hline Center & Atomic & \multirow{2}{*}{$\begin{array}{c}\text { Atomic } \\
\text { Number }\end{array}$} & Number & Type & \multicolumn{2}{|c|}{ Coordinates (Angstroms) } \\
\cline { 4 - 6 } & & $\mathrm{X}$ & $\mathrm{Y}$ & $\mathrm{Z}$ \\
\hline 1 & 6 & 0 & 0.020434 & -0.46751 & 0.054539 \\
\hline 2 & 6 & 0 & -1.38956 & -0.0416 & -0.3224 \\
\hline 3 & 1 & 0 & -1.38041 & 0.142607 & -1.40718 \\
\hline 4 & 1 & 0 & 0.294876 & -1.41634 & -0.4081 \\
\hline 5 & 1 & 0 & 0.134717 & -0.56133 & 1.135797 \\
\hline 6 & 6 & 0 & -1.79366 & 1.254301 & 0.379168 \\
\hline
\end{tabular}




\begin{tabular}{|c|c|c|c|c|c|}
\hline 7 & 6 & 0 & -2.33948 & -1.15631 & -0.02597 \\
\hline 8 & 1 & 0 & -2.79834 & 1.552534 & 0.078545 \\
\hline 9 & 1 & 0 & -1.7905 & 1.115524 & 1.461933 \\
\hline 10 & 1 & 0 & -1.10691 & 2.061726 & 0.130722 \\
\hline 11 & 1 & 0 & -3.36162 & -0.9441 & 0.249011 \\
\hline 12 & 1 & 0 & -2.08042 & -2.1797 & -0.25676 \\
\hline 13 & 8 & 0 & 0.927128 & 0.561416 & -0.42589 \\
\hline 14 & 6 & 0 & 2.230769 & 0.339456 & -0.16137 \\
\hline 15 & 8 & 0 & 2.684429 & -0.62459 & 0.430341 \\
\hline 16 & 1 & 0 & 2.825102 & 1.164437 & -0.56343 \\
\hline
\end{tabular}

\begin{tabular}{|c|c|c|c|c|c|}
\hline \multicolumn{7}{|c|}{ P8 } \\
\hline Center & Atomic & Atomic & \multicolumn{2}{|c|}{ Coordinates (Angstroms) } \\
\cline { 4 - 6 } Number & Number & Type & $\mathrm{X}$ & $\mathrm{Y}$ & $\mathrm{Z}$ \\
\hline 1 & 6 & 0 & 0.020428 & -0.46757 & 0.054547 \\
\hline 2 & 6 & 0 & -1.3896 & -0.04159 & -0.32237 \\
\hline 3 & 1 & 0 & -1.38034 & 0.142792 & -1.40711 \\
\hline 4 & 1 & 0 & 0.294684 & -1.4166 & -0.4078 \\
\hline 5 & 1 & 0 & 0.134844 & -0.56104 & 1.135822 \\
\hline 6 & 6 & 0 & -1.7936 & 1.254352 & 0.379336 \\
\hline 7 & 6 & 0 & -2.33962 & -1.15618 & -0.02591 \\
\hline 8 & 1 & 0 & -2.79821 & 1.552753 & 0.078616 \\
\hline 9 & 1 & 0 & -1.79057 & 1.115474 & 1.462086 \\
\hline 10 & 1 & 0 & -1.10672 & 2.061695 & 0.131013 \\
\hline 11 & 1 & 0 & -3.36183 & -0.94382 & 0.248788 \\
\hline 12 & 1 & 0 & -2.081 & -2.17955 & -0.25726 \\
\hline 13 & 8 & 0 & 0.927196 & 0.561081 & -0.42632 \\
\hline 14 & 6 & 0 & 2.230833 & 0.339352 & -0.16155 \\
\hline 15 & 8 & 0 & 2.684466 & -0.62434 & 0.430756 \\
\hline 16 & 1 & 0 & 2.825189 & 1.164129 & -0.564 \\
\hline
\end{tabular}

\begin{tabular}{|c|c|c|c|c|c|}
\hline \multicolumn{5}{|c|}{ P9 } \\
\hline Center & Atomic & Atomic & \multicolumn{3}{|c|}{ Coordinates (Angstroms) } \\
\cline { 4 - 6 } Number & Number & Type & X & Y & Z \\
\hline
\end{tabular}




\begin{tabular}{|c|c|c|c|c|c|}
\hline 1 & 6 & 0 & 0.017243 & -0.48764 & -0.01052 \\
\hline 2 & 6 & 0 & -1.39483 & -0.0349 & -0.34515 \\
\hline 3 & 1 & 0 & -1.43114 & 0.173891 & -1.41813 \\
\hline 4 & 1 & 0 & 0.292093 & -1.38988 & -0.55655 \\
\hline 5 & 1 & 0 & 0.131274 & -0.67917 & 1.058707 \\
\hline 6 & 6 & 0 & -1.75605 & 1.253576 & 0.41199 \\
\hline 7 & 6 & 0 & -2.35171 & -1.13974 & -0.03033 \\
\hline 8 & 1 & 0 & -2.76737 & 1.57089 & 0.159751 \\
\hline 9 & 1 & 0 & -1.71267 & 1.082866 & 1.489326 \\
\hline 10 & 1 & 0 & -1.06433 & 2.055911 & 0.158321 \\
\hline 11 & 1 & 0 & -2.2916 & -1.65141 & 0.920409 \\
\hline 12 & 1 & 0 & -3.23122 & -1.30071 & -0.63412 \\
\hline 13 & 8 & 0 & 0.924244 & 0.581237 & -0.39329 \\
\hline 14 & 6 & 0 & 2.226002 & 0.341941 & -0.13468 \\
\hline 15 & 8 & 0 & 2.676916 & -0.66357 & 0.385841 \\
\hline 16 & 1 & 0 & 2.821759 & 1.196796 & -0.46607 \\
\hline
\end{tabular}

\begin{tabular}{|c|c|c|c|c|c|}
\hline \multicolumn{7}{|c|}{ P10 } \\
\hline Center & Atomic & Atomic & \multicolumn{2}{|c|}{ Coordinates (Angstroms) } \\
\cline { 4 - 6 } Number & Number & Type & $\mathrm{X}$ & $\mathrm{Y}$ & $\mathrm{Z}$ \\
\hline 1 & 6 & 0 & 0.09532 & -0.43963 & 0.021331 \\
\hline 2 & 6 & 0 & -1.30707 & 0.002096 & -0.33668 \\
\hline 3 & 1 & 0 & -1.33295 & 0.158289 & -1.41952 \\
\hline 4 & 1 & 0 & 0.369446 & -1.37746 & -0.46322 \\
\hline 5 & 1 & 0 & 0.227523 & -0.5412 & 1.100672 \\
\hline 6 & 6 & 0 & -1.6759 & 1.307657 & 0.361694 \\
\hline 7 & 6 & 0 & -2.2856 & -1.11739 & 0.020133 \\
\hline 8 & 1 & 0 & -2.68114 & 1.620226 & 0.077512 \\
\hline 9 & 1 & 0 & -1.65885 & 1.175968 & 1.446265 \\
\hline 10 & 1 & 0 & -0.98248 & 2.106003 & 0.10195 \\
\hline 11 & 1 & 0 & -2.26745 & -1.3183 & 1.093433 \\
\hline 12 & 1 & 0 & -3.30224 & -0.8301 & -0.24768 \\
\hline 13 & 1 & 0 & -2.04599 & -2.04312 & -0.50472 \\
\hline 14 & 8 & 0 & 1.026451 & 0.597535 & -0.4522 \\
\hline 15 & 6 & 0 & 2.306619 & 0.364821 & -0.18256 \\
\hline 16 & 8 & 0 & 2.832781 & -0.55449 & 0.39117 \\
\hline
\end{tabular}




\begin{tabular}{|c|c|c|c|c|c|}
\hline \multicolumn{6}{|c|}{ HCl } \\
\hline Center & Atomic & Atomic & \multicolumn{3}{|c|}{ Coordinates (Angstroms) } \\
\cline { 4 - 6 } Number & Number & Type & $\mathrm{X}$ & $\mathrm{Y}$ & $\mathrm{Z}$ \\
\hline 1 & 17 & 0 & 0 & 0 & 0.070556 \\
\hline 2 & 1 & 0 & 0 & 0 & -1.19946 \\
\hline
\end{tabular}

Table S5: Branching ratios for the reactions of isopropyl formate with $\mathrm{Cl}$ atoms (R1) in the temperature range of $200-400 \mathrm{~K}$.

\begin{tabular}{|c|c|c|c|}
\hline \multirow{2}{*}{ Temperature(K) } & \multicolumn{3}{|c|}{ Branching Ratio(\%) } \\
\cline { 2 - 4 } & $-\mathrm{CH} 3$ & $-\mathrm{CH}$ & $-\mathrm{HC}(\mathrm{O}) \mathrm{O}$ \\
\hline 200 & 1.56 & 51.59 & 46.86 \\
\hline 225 & 1.85 & 50.08 & 48.08 \\
\hline 250 & 2.10 & 50.19 & 47.70 \\
\hline 275 & 2.50 & 53.13 & 44.37 \\
\hline $\mathbf{2 9 8}$ & $\mathbf{2 . 7 9}$ & $\mathbf{5 6 . 1 3}$ & $\mathbf{4 1 . 0 9}$ \\
\hline 300 & 2.97 & 56.24 & 40.79 \\
\hline 325 & 3.32 & 55.67 & 41.01 \\
\hline 350 & 3.70 & 55.92 & 40.38 \\
\hline 375 & 4.39 & 60.17 & 35.43 \\
\hline 400 & 5.01 & 56.62 & 29.26 \\
\hline & & & \\
\hline
\end{tabular}


Table S6: Branching ratios for the reactions of isobutyl formate with $\mathrm{Cl}$ atoms (R2) in the temperature range of $200-400 \mathrm{~K}$.

\begin{tabular}{|c|c|c|c|c|}
\hline \multirow{2}{*}{ Temperature(K) } & \multicolumn{4}{|c|}{ Branching Ratio(\%) } \\
\cline { 2 - 5 } & $-\mathrm{CH}_{3}$ & $-\mathrm{CH}$ & $-\mathrm{CH}_{2}$ & $-\mathrm{HC}(\mathrm{O}) \mathrm{O}$ \\
\hline 200 & $4.27 \times 10^{-02}$ & 98.48 & 0.62 & 0.85 \\
\hline 225 & $7.32 \times 10^{-02}$ & 97.59 & 1.01 & 1.32 \\
\hline 250 & $1.16 \times 10^{-01}$ & 96.92 & 1.06 & 1.91 \\
\hline 275 & $2.24 \times 10^{-01}$ & 96.23 & 0.96 & 2.58 \\
\hline $\mathbf{2 9 8}$ & $3.10 \times 10^{-01}$ & $\mathbf{9 5 . 2 3}$ & $\mathbf{1 . 1 9}$ & $\mathbf{3 . 2 7}$ \\
\hline 300 & $3.18 \times 10^{-01}$ & 95.14 & 1.21 & 3.34 \\
\hline 325 & $4.35 \times 10^{-01}$ & 93.97 & 1.46 & 4.13 \\
\hline 350 & $5.77 \times 10^{-01}$ & 92.70 & 1.72 & 5.00 \\
\hline 375 & $7.95 \times 10^{-01}$ & 91.36 & 1.96 & 5.88 \\
\hline 400 & 1.01 & 90.00 & 2.21 & 6.78 \\
\hline
\end{tabular}

Table S7: CVT and CVT/SCT rate coefficients for the reaction of isopropyl formate with $\mathrm{Cl}$ atom in the temperature range of $200-400 \mathrm{~K}$ at CCSD(T)/cc-pVDZ//MP2/6-31+G(d, p) level of theory.

\begin{tabular}{|c|c|c|c|c|c|c|c|c|}
\hline \multirow{2}{*}{$\begin{array}{c}\text { Temp } \\
(K)\end{array}$} & \multicolumn{2}{|c|}{ TS1 } & \multicolumn{2}{c|}{ TS2 } & \multicolumn{2}{c|}{ TS3 } & \multicolumn{2}{c|}{ TS4 } \\
\cline { 2 - 9 } & CVT & CVT/SCT & CVT & CVT/SCT & CVT & CVT/SCT & CVT & CVT/SCT \\
\hline 200 & $7.37 \times 10^{-12}$ & $1.08 \times 10^{-11}$ & $4.75 \times 10^{-18}$ & $1.09 \times 10^{-16}$ & $3.64 \times 10^{-19}$ & $1.51 \times 10^{-16}$ & $3.60 \times 10^{-17}$ & $2.46 \times 10^{-15}$ \\
\hline 225 & $7.33 \times 10^{-12}$ & $1 \times 10^{-11}$ & $7.17 \times 10^{-18}$ & $1.31 \times 10^{-16}$ & $5.42 \times 10^{-19}$ & $1.76 \times 10^{-16}$ & $7.67 \times 10^{-17}$ & $2.41 \times 10^{-15}$ \\
\hline 250 & $7.47 \times 10^{-12}$ & $9.69 \times 10^{-12}$ & $1.05 \times 10^{-17}$ & $1.57 \times 10^{-16}$ & $8.18 \times 10^{-19}$ & $2.05 \times 10^{-16}$ & $1.39 \times 10^{-16}$ & $2.44 \times 10^{-15}$ \\
\hline 275 & $7.74 \times 10^{-12}$ & $9.63 \times 10^{-12}$ & $7.20 \times 10^{-18}$ & $8.96 \times 10^{-17}$ & $1.24 \times 10^{-18}$ & $2.36 \times 10^{-16}$ & $2.23 \times 10^{-16}$ & $2.51 \times 10^{-15}$ \\
\hline 298 & $8.03 \times 10^{-12}$ & $9.74 \times 10^{-12}$ & $9.89 \times 10^{-18}$ & $1.06 \times 10^{-16}$ & $1.82 \times 10^{-18}$ & $2.68 \times 10^{-16}$ & $3.19 \times 10^{-16}$ & $2.60 \times 10^{-15}$ \\
\hline 300 & $8.1 \times 10^{-12}$ & $9.76 \times 10^{-12}$ & $1.02 \times 10^{-17}$ & $1.07 \times 10^{-16}$ & $1.88 \times 10^{-18}$ & $2.71 \times 10^{-16}$ & $3.28 \times 10^{-16}$ & $2.61 \times 10^{-15}$ \\
\hline 325 & $5.57 \times 10^{-12}$ & $6.53 \times 10^{-12}$ & $1.40 \times 10^{-17}$ & $1.27 \times 10^{-16}$ & $2.82 \times 10^{-18}$ & $3.09 \times 10^{-16}$ & $4.55 \times 10^{-16}$ & $2.75 \times 10^{-15}$ \\
\hline 350 & $5.86 \times 10^{-12}$ & $6.73 \times 10^{-12}$ & $1.89 \times 10^{-17}$ & $1.49 \times 10^{-16}$ & $4.17 \times 10^{-18}$ & $3.52 \times 10^{-16}$ & $6.02 \times 10^{-16}$ & $2.91 \times 10^{-15}$ \\
\hline 375 & $6.19 \times 10^{-12}$ & $6.98 \times 10^{-12}$ & $2.49 \times 10^{-17}$ & $1.74 \times 10^{-16}$ & $6.07 \times 10^{-18}$ & $3.98 \times 10^{-16}$ & $7.65 \times 10^{-16}$ & $3.10 \times 10^{-15}$ \\
\hline 400 & $6.53 \times 10^{-12}$ & $7.27 \times 10^{-12}$ & $3.24 \times 10^{-17}$ & $2.01 \times 10^{-16}$ & $8.65 \times 10^{-18}$ & $4.49 \times 10^{-16}$ & $9.53 \times 10^{-16}$ & $3.31 \times 10^{-15}$ \\
\hline
\end{tabular}

\begin{tabular}{|c|c|c|c|c|}
\hline $\begin{array}{c}\text { Temp } \\
(\mathrm{K})\end{array}$ & TS5 & TS6 & TS7 & TS8 \\
\hline
\end{tabular}




\begin{tabular}{|c|c|c|c|c|c|c|c|c|}
\cline { 1 - 5 } & CVT & CVT/SCT & CVT & CVT/SCT & CVT & CVT/SCT & CVT & CVT/SCT \\
\hline 200 & $8.35 \times 10^{-19}$ & $1.37 \times 10^{-16}$ & $5.01 \times 10^{-14}$ & $2.75 \times 10^{-13}$ & $3.00 \times 10^{-16}$ & $4.82 \times 10^{-14}$ & $6.69 \times 10^{-12}$ & $9.81 \times 10^{-12}$ \\
\hline 225 & $1.23 \times 10^{-18}$ & $1.58 \times 10^{-16}$ & $6.04 \times 10^{-14}$ & $3.12 \times 10^{-13}$ & $4.06 \times 10^{-16}$ & $5.37 \times 10^{-14}$ & $7.03 \times 10^{-12}$ & $9.6 \times 10^{-12}$ \\
\hline 250 & $1.84 \times 10^{-18}$ & $1.81 \times 10^{-16}$ & $7.23 \times 10^{-14}$ & $3.52 \times 10^{-13}$ & $5.61 \times 10^{-16}$ & $5.97 \times 10^{-14}$ & $7.25 \times 10^{-12}$ & $9.4 \times 10^{-12}$ \\
\hline 275 & $2.74 \times 10^{-18}$ & $2.07 \times 10^{-16}$ & $8.59 \times 10^{-14}$ & $3.94 \times 10^{-13}$ & $7.84 \times 10^{-16}$ & $6.63 \times 10^{-14}$ & $6.54 \times 10^{-12}$ & $8.21 \times 10^{-12}$ \\
\hline 298 & $3.93 \times 10^{-18}$ & $2.33 \times 10^{-16}$ & $1.00 \times 10^{-13}$ & $4.34 \times 10^{-13}$ & $1.07 \times 10^{-15}$ & $7.28 \times 10^{-14}$ & $5.73 \times 10^{-12}$ & $7.13 \times 10^{-12}$ \\
\hline 300 & $4.06 \times 10^{-18}$ & $2.35 \times 10^{-16}$ & $1.01 \times 10^{-13}$ & $4.37 \times 10^{-13}$ & $1.10 \times 10^{-15}$ & $7.34 \times 10^{-14}$ & $5.73 \times 10^{-12}$ & $7.05 \times 10^{-12}$ \\
\hline 325 & $5.91 \times 10^{-18}$ & $2.66 \times 10^{-16}$ & $1.19 \times 10^{-13}$ & $4.83 \times 10^{-13}$ & $1.53 \times 10^{-15}$ & $8.12 \times 10^{-14}$ & $5.79 \times 10^{-12}$ & $7.02 \times 10^{-12}$ \\
\hline 350 & $8.46 \times 10^{-18}$ & $3.00 \times 10^{-16}$ & $1.39 \times 10^{-13}$ & $5.30 \times 10^{-13}$ & $2.12 \times 10^{-15}$ & $8.96 \times 10^{-14}$ & $5.65 \times 10^{-12}$ & $6.81 \times 10^{-12}$ \\
\hline 375 & $1.19 \times 10^{-17}$ & $3.37 \times 10^{-16}$ & $1.61 \times 10^{-13}$ & $5.79 \times 10^{-13}$ & $2.89 \times 10^{-15}$ & $9.86 \times 10^{-14}$ & $4.65 \times 10^{-12}$ & $5.5 \times 10^{-12}$ \\
\hline 400 & $1.63 \times 10^{-17}$ & $3.77 \times 10^{-16}$ & $1.85 \times 10^{-13}$ & $6.30 \times 10^{-13}$ & $3.87 \times 10^{-15}$ & $1.08 \times 10^{-13}$ & $4.09 \times 10^{-12}$ & $4.79 \times 10^{-12}$ \\
\hline
\end{tabular}

Table S8: CVT and CVT/SCT rate coefficients for the reaction of isobutyl formate with $\mathrm{Cl}$ atom in the temperature range of $200-400 \mathrm{~K}$ at $\mathrm{CCSD}(\mathrm{T}) / \mathrm{cc}-\mathrm{pVDZ} / \mathrm{MP} 2 / 6-31+\mathrm{G}(\mathrm{d}, \mathrm{p})$ level of theory.

\begin{tabular}{|c|c|c|c|c|c|c|c|c|c|c|}
\hline \multirow{2}{*}{$\begin{array}{c}\text { Temp } \\
(K)\end{array}$} & \multicolumn{2}{|c|}{ TS1 } & \multicolumn{2}{c|}{ TS2 } & \multicolumn{2}{c|}{ TS3 } & \multicolumn{2}{c|}{ TS4 } & \multicolumn{2}{c|}{ TS5 } \\
\cline { 2 - 11 } & CVT & CVT/SCT & CVT & CVT/SCT & CVT & CVT/SCT & CVT & CVT/SCT & CVT & CVT/SCT \\
\hline 200 & $1.87 \times 10^{-11}$ & $1.31 \times 10^{-10}$ & $1.07 \times 10^{-13}$ & $1.90 \times 10^{-13}$ & $1.05 \times 10^{-13}$ & $6.41 \times 10^{-13}$ & $2.38 \times 10^{-15}$ & $7.04 \times 10^{-15}$ & $1.71 \times 10^{-16}$ & $1.56 \times 10^{-15}$ \\
\hline 225 & $2.32 \times 10^{-11}$ & $9.51 \times 10^{-11}$ & $1.41 \times 10^{-13}$ & $2.29 \times 10^{-13}$ & $1.45 \times 10^{-13}$ & $7.54 \times 10^{-13}$ & $3.24 \times 10^{-15}$ & $8.60 \times 10^{-15}$ & $2.68 \times 10^{-16}$ & $1.79 \times 10^{-15}$ \\
\hline 250 & $2.6 \times 10^{-11}$ & $7.47 \times 10^{-11}$ & $1.77 \times 10^{-13}$ & $2.69 \times 10^{-13}$ & $1.22 \times 10^{-13}$ & $5.46 \times 10^{-13}$ & $4.36 \times 10^{-15}$ & $1.04 \times 10^{-14}$ & $4.24 \times 10^{-16}$ & $2.12 \times 10^{-15}$ \\
\hline 275 & $2.76 \times 10^{-11}$ & $6.22 \times 10^{-11}$ & $1.36 \times 10^{-14}$ & $1.95 \times 10^{-14}$ & $1.54 \times 10^{-13}$ & $6.03 \times 10^{-13}$ & $5.77 \times 10^{-15}$ & $1.26 \times 10^{-14}$ & $6.59 \times 10^{-16}$ & $2.56 \times 10^{-15}$ \\
\hline 298 & $2.82 \times 10^{-11}$ & $5.44 \times 10^{-11}$ & $1.65 \times 10^{-14}$ & $2.25 \times 10^{-14}$ & $1.87 \times 10^{-13}$ & $6.57 \times 10^{-13}$ & $7.36 \times 10^{-15}$ & $1.49 \times 10^{-14}$ & $9.66 \times 10^{-16}$ & $3.07 \times 10^{-15}$ \\
\hline 300 & $2.82 \times 10^{-11}$ & $5.39 \times 10^{-11}$ & $1.67 \times 10^{-14}$ & $2.28 \times 10^{-14}$ & $1.90 \times 10^{-13}$ & $6.62 \times 10^{-13}$ & $7.51 \times 10^{-15}$ & $1.51 \times 10^{-14}$ & $9.97 \times 10^{-16}$ & $3.13 \times 10^{-15}$ \\
\hline 325 & $2.85 \times 10^{-11}$ & $4.82 \times 10^{-11}$ & $2.03 \times 10^{-14}$ & $2.66 \times 10^{-14}$ & $2.32 \times 10^{-13}$ & $7.23 \times 10^{-13}$ & $9.63 \times 10^{-15}$ & $1.80 \times 10^{-14}$ & $1.46 \times 10^{-15}$ & $3.85 \times 10^{-15}$ \\
\hline 350 & $2.86 \times 10^{-11}$ & $4.41 \times 10^{-11}$ & $2.43 \times 10^{-14}$ & $3.09 \times 10^{-14}$ & $2.77 \times 10^{-13}$ & $7.86 \times 10^{-13}$ & $1.22 \times 10^{-14}$ & $2.13 \times 10^{-14}$ & $2.08 \times 10^{-15}$ & $4.75 \times 10^{-15}$ \\
\hline 375 & $2.86 \times 10^{-11}$ & $4.12 \times 10^{-11}$ & $2.88 \times 10^{-14}$ & $3.56 \times 10^{-14}$ & $3.27 \times 10^{-13}$ & $8.50 \times 10^{-13}$ & $1.51 \times 10^{-14}$ & $2.51 \times 10^{-14}$ & $2.88 \times 10^{-15}$ & $5.85 \times 10^{-15}$ \\
\hline 400 & $2.85 \times 10^{-11}$ & $3.9 \times 10^{-11}$ & $3.37 \times 10^{-14}$ & $4.08 \times 10^{-14}$ & $3.81 \times 10^{-13}$ & $9.17 \times 10^{-13}$ & $1.86 \times 10^{-14}$ & $2.94 \times 10^{-14}$ & $3.89 \times 10^{-15}$ & $7.18 \times 10^{-15}$ \\
\hline
\end{tabular}




\begin{tabular}{|c|c|c|c|c|c|c|c|c|c|c|}
\hline \multirow{2}{*}{$\begin{array}{c}\text { Temp } \\
(K\end{array}$} & \multicolumn{2}{|c|}{ TS6 } & \multicolumn{2}{c|}{ TS7 } & \multicolumn{2}{c|}{ TS8 } & \multicolumn{3}{c|}{ TS10 } & \multicolumn{2}{c|}{ TS10 } \\
\cline { 2 - 11 } & CVT & CVT/SCT & CVT & CVT/SCT & CVT & CVT/SCT & CVT & CVT/SCT & CVT & CVT/SCT \\
\hline 200 & $1.37 \times 10^{-16}$ & $5.77 \times 10^{-16}$ & $7.71 \times 10^{-16}$ & $2.41 \times 10^{-15}$ & $1.21 \times 10^{-14}$ & $4.36 \times 10^{-14}$ & $3.03 \times 10^{-16}$ & $1.61 \times 10^{-15}$ & $1.61 \times 10^{-13}$ & $1.13 \times 10^{-12}$ \\
\hline 225 & $2.31 \times 10^{-16}$ & $7.71 \times 10^{-16}$ & $1.13 \times 10^{-15}$ & $3.04 \times 10^{-15}$ & $1.77 \times 10^{-14}$ & $5.51 \times 10^{-14}$ & $4.64 \times 10^{-16}$ & $2.03 \times 10^{-15}$ & $3.15 \times 10^{-13}$ & $1.29 \times 10^{-12}$ \\
\hline 250 & $3.81 \times 10^{-16}$ & $1.03 \times 10^{-15}$ & $1.63 \times 10^{-15}$ & $3.84 \times 10^{-15}$ & $2.57 \times 10^{-14}$ & $6.93 \times 10^{-14}$ & $7.06 \times 10^{-16}$ & $2.55 \times 10^{-15}$ & $5.11 \times 10^{-13}$ & $1.47 \times 10^{-12}$ \\
\hline 275 & $6.06 \times 10^{-16}$ & $1.38 \times 10^{-15}$ & $2.30 \times 10^{-15}$ & $4.83 \times 10^{-15}$ & $3.65 \times 10^{-14}$ & $8.68 \times 10^{-14}$ & $1.21 \times 10^{-14}$ & $3.68 \times 10^{-14}$ & $7.41 \times 10^{-13}$ & $1.67 \times 10^{-12}$ \\
\hline 298 & $8.97 \times 10^{-16}$ & $1.80 \times 10^{-15}$ & $3.10 \times 10^{-15}$ & $5.93 \times 10^{-15}$ & $4.95 \times 10^{-14}$ & $1.06 \times 10^{-13}$ & $1.71 \times 10^{-14}$ & $4.52 \times 10^{-14}$ & $9.7 \times 10^{-13}$ & $1.87 \times 10^{-12}$ \\
\hline 300 & $9.26 \times 10^{-16}$ & $1.84 \times 10^{-15}$ & $3.17 \times 10^{-15}$ & $6.04 \times 10^{-15}$ & $5.08 \times 10^{-14}$ & $1.08 \times 10^{-13}$ & $1.76 \times 10^{-14}$ & $4.60 \times 10^{-14}$ & $9.91 \times 10^{-13}$ & $1.89 \times 10^{-12}$ \\
\hline 325 & $1.37 \times 10^{-15}$ & $2.43 \times 10^{-15}$ & $4.29 \times 10^{-15}$ & $7.50 \times 10^{-15}$ & $6.91 \times 10^{-14}$ & $1.34 \times 10^{-13}$ & $2.49 \times 10^{-14}$ & $5.74 \times 10^{-14}$ & $1.25 \times 10^{-12}$ & $2.12 \times 10^{-12}$ \\
\hline 350 & $1.95 \times 10^{-15}$ & $3.18 \times 10^{-15}$ & $5.67 \times 10^{-15}$ & $9.25 \times 10^{-15}$ & $9.21 \times 10^{-14}$ & $1.65 \times 10^{-13}$ & $3.45 \times 10^{-14}$ & $7.12 \times 10^{-14}$ & $1.54 \times 10^{-12}$ & $2.38 \times 10^{-12}$ \\
\hline 375 & $1.82 \times 10^{-14}$ & $2.76 \times 10^{-14}$ & $7.35 \times 10^{-15}$ & $1.13 \times 10^{-14}$ & $1.20 \times 10^{-13}$ & $2.01 \times 10^{-13}$ & $4.67 \times 10^{-14}$ & $8.78 \times 10^{-14}$ & $1.84 \times 10^{-12}$ & $2.65 \times 10^{-12}$ \\
\hline 400 & $2.44 \times 10^{-14}$ & $3.50 \times 10^{-14}$ & $9.37 \times 10^{-15}$ & $1.37 \times 10^{-14}$ & $1.54 \times 10^{-13}$ & $2.44 \times 10^{-13}$ & $6.18 \times 10^{-14}$ & $1.08 \times 10^{-13}$ & $2.15 \times 10^{-12}$ & $2.94 \times 10^{-12}$ \\
\hline
\end{tabular}

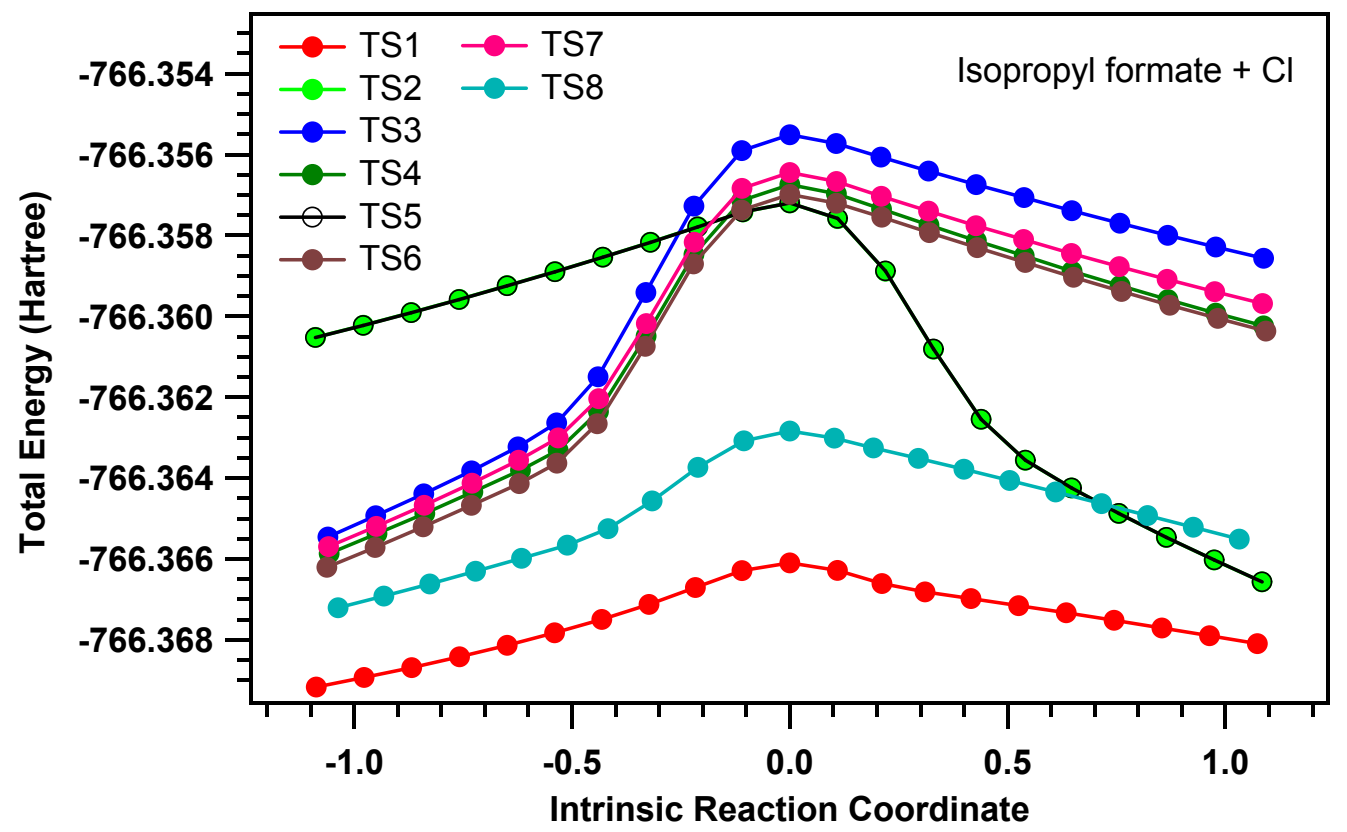

Figure S1: IRC plots for the transition states (TS1, TS2, TS3, TS4, TS5, TS6, TS7 and TS8) obtained for the reaction of isopropyl formate with $\mathrm{Cl}$ atoms. 


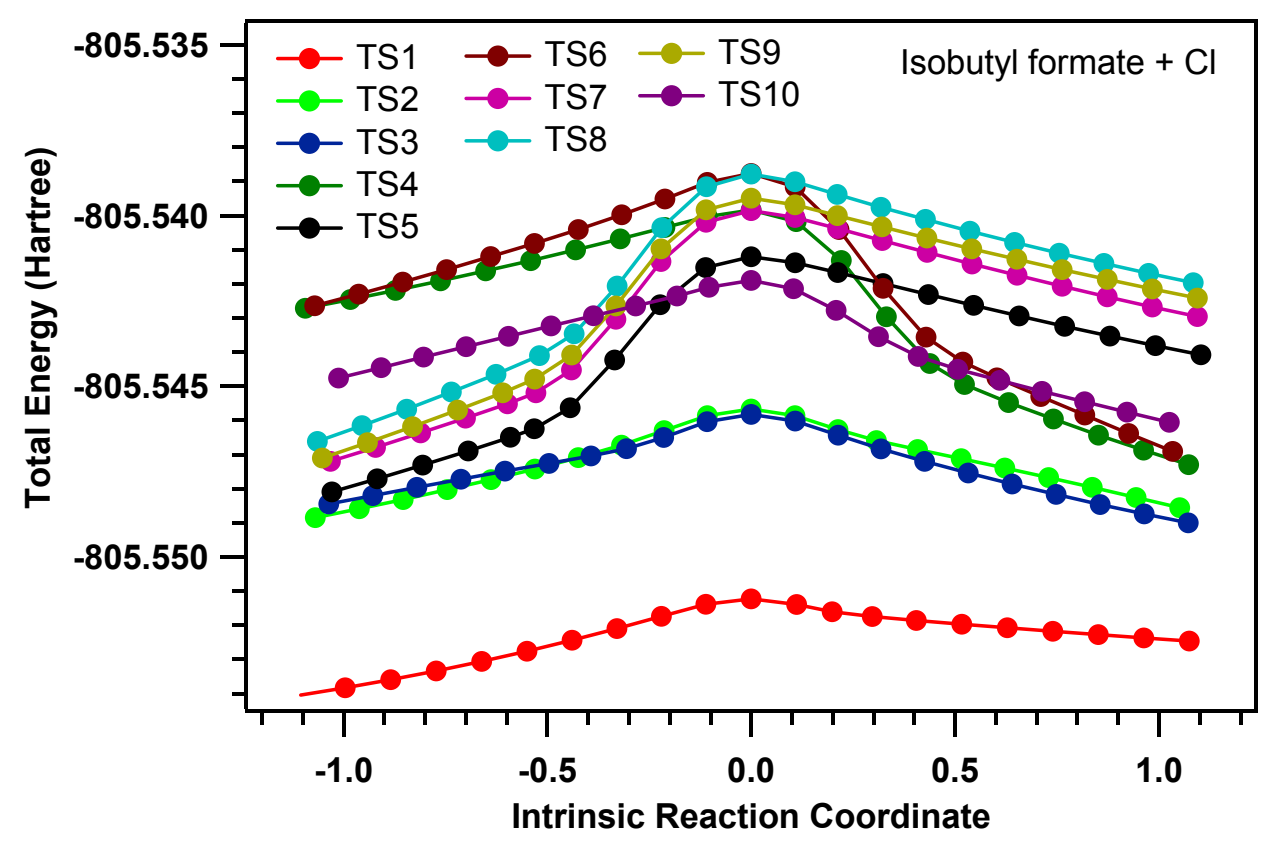

Figure S2: IRC plots for the transition states (TS1, TS2, TS3, TS4, TS5, TS6, TS7, TS8, TS9 and TS10) obtained for the reaction of isobutyl formate with $\mathrm{Cl}$ atoms. 


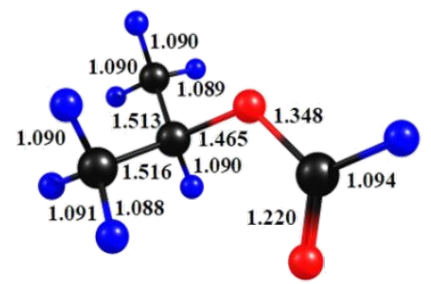

Isopropyl formate

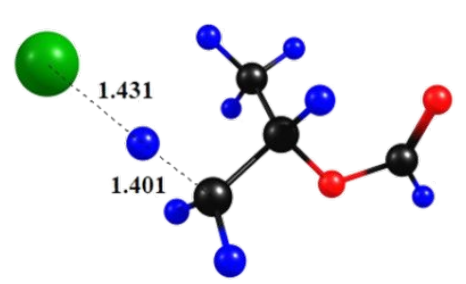

TS3
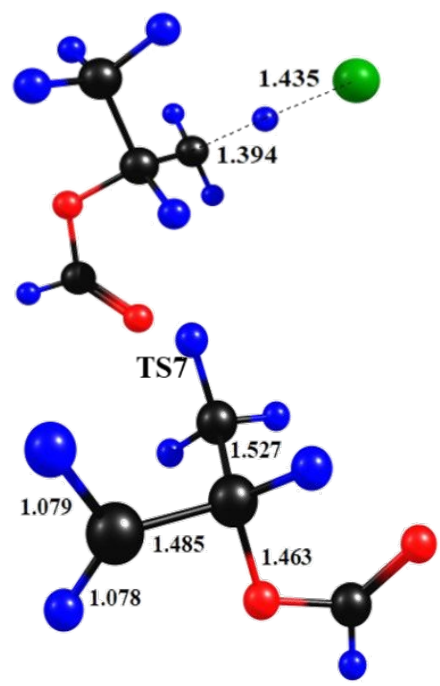

P2

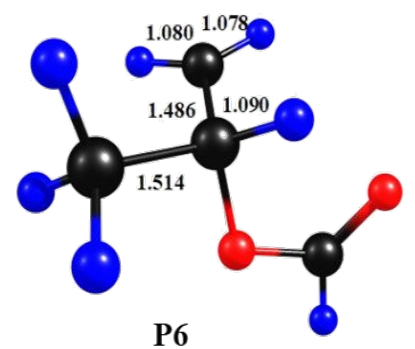

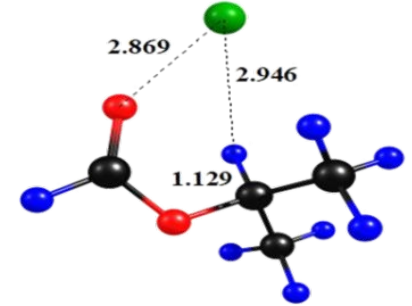

RC1

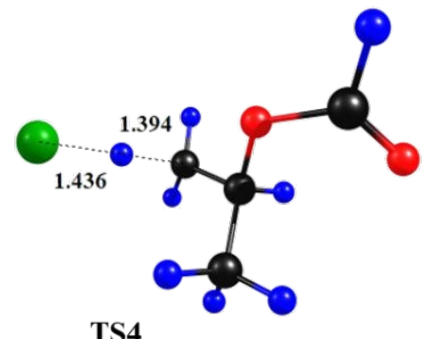

TS4
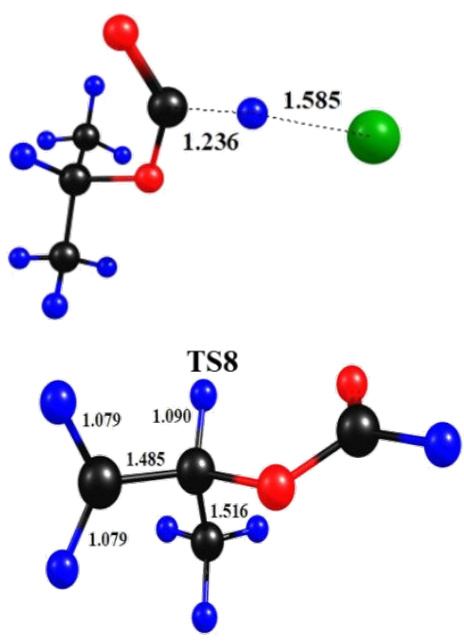

P3

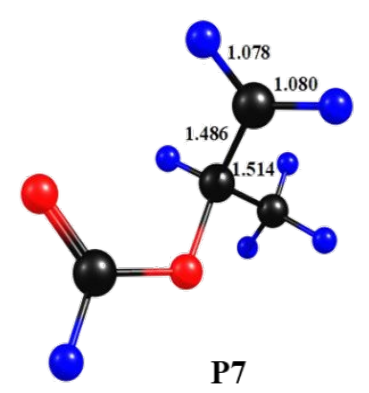

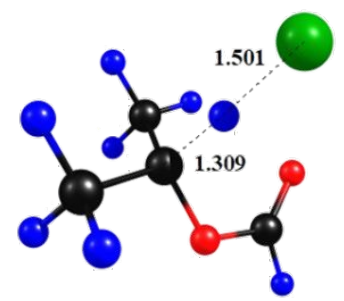

TS1
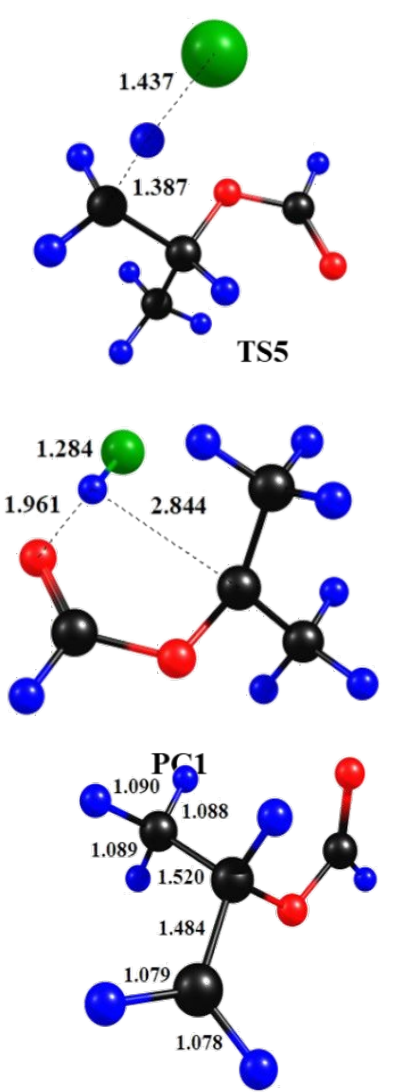

P4

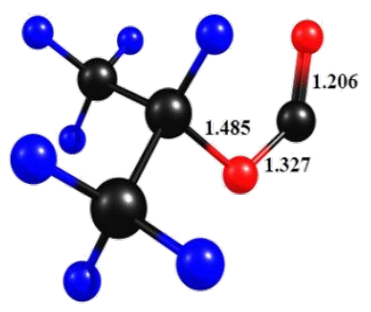

P8

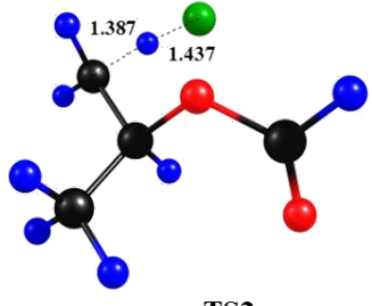

TS2
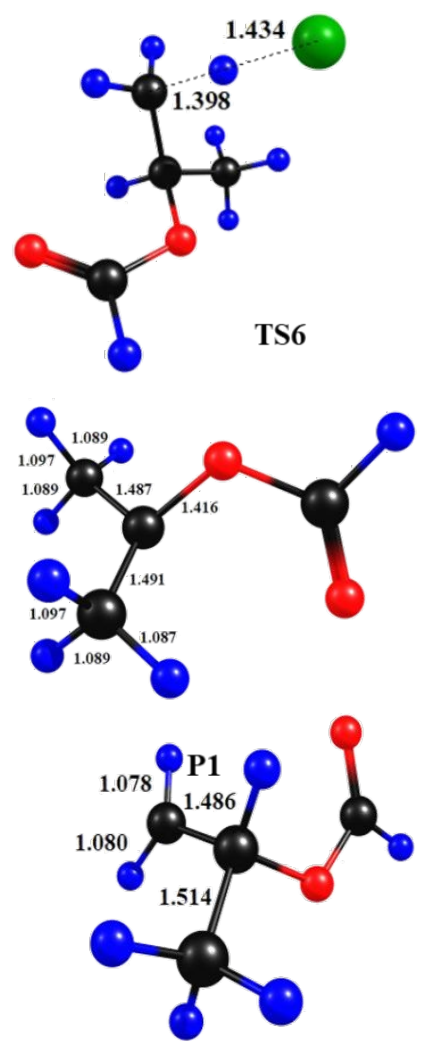

P5

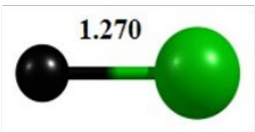

HCl 
Figure S3: Optimized geometries of the reactants, pre-reactive complexes, transition states and products for the reaction of $\mathrm{Cl}$ atoms with isopropyl formate obtained at $\mathrm{MP} 2 / 6-31+\mathrm{G}(\mathrm{d}, \mathrm{p})$ level of theory. Black color represents carbon atoms, blue color represents hydrogen atoms, green color represents $\mathrm{Cl}$ atoms and red color represents oxygen atoms. 


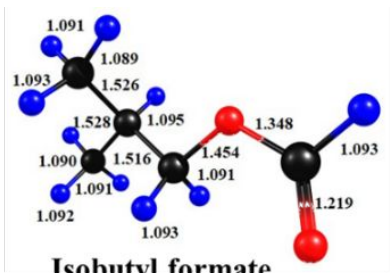

Isobutyl formate

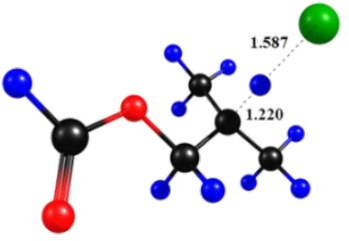

TS1

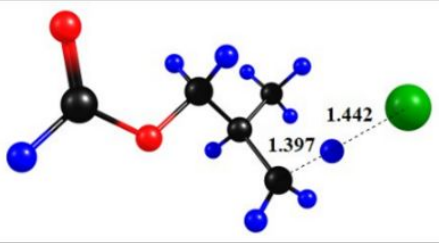

TS5

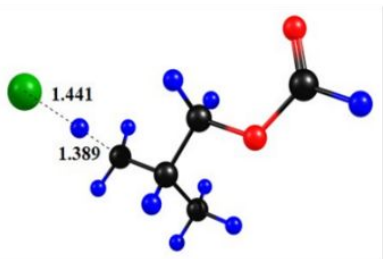

TS9
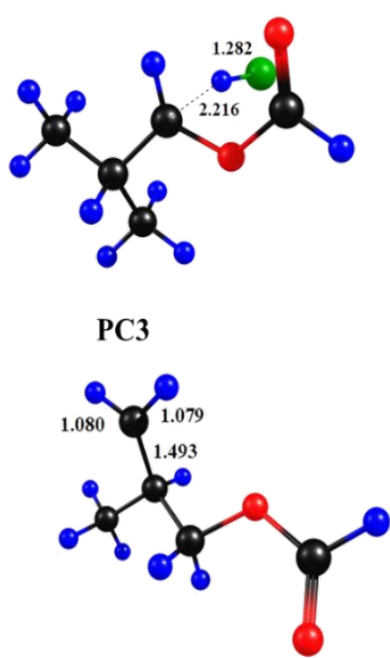

P4
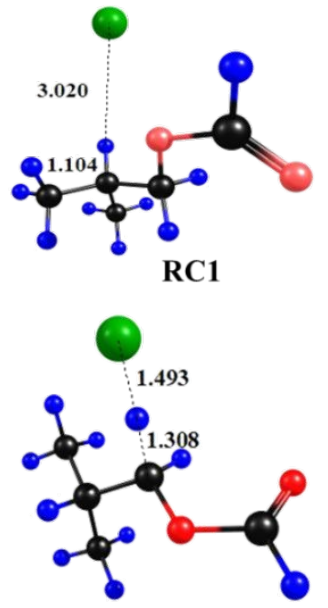

TS2

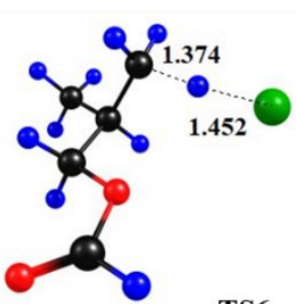

TS6

PC1

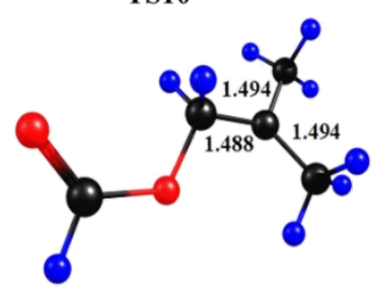

P1

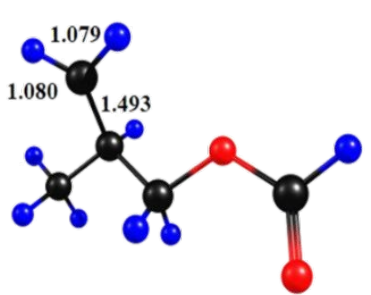

P5

RC2
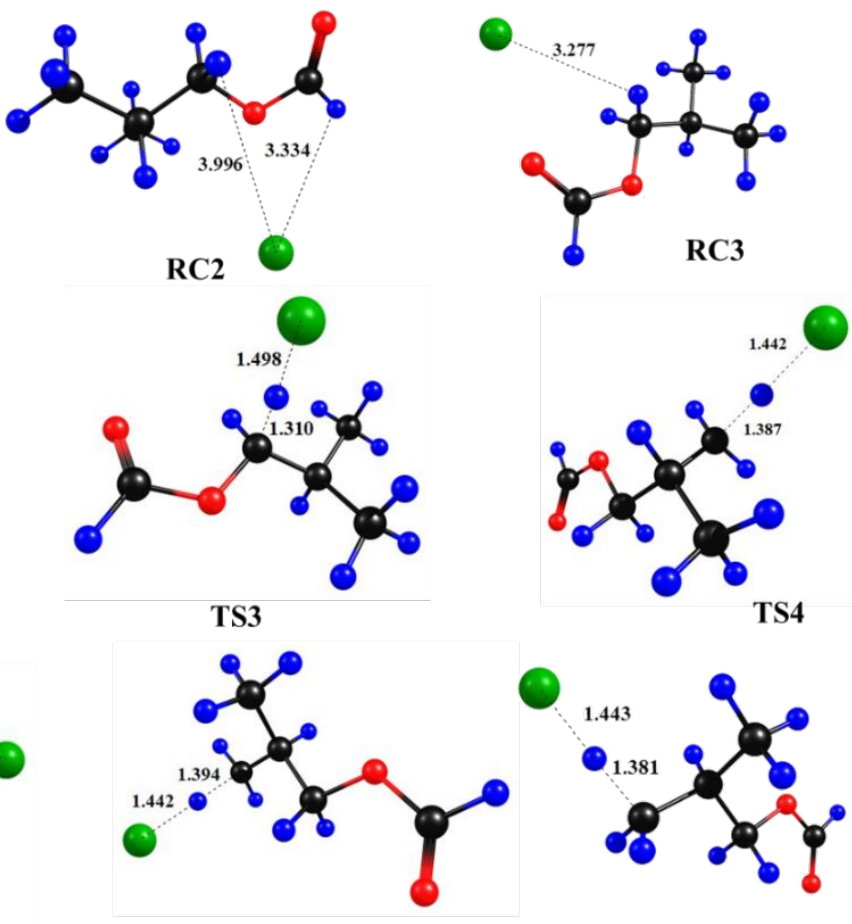

TS7

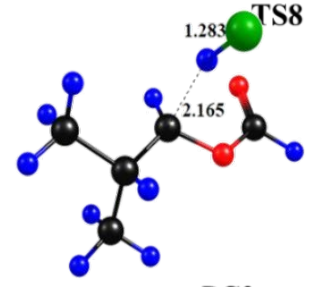

PC2

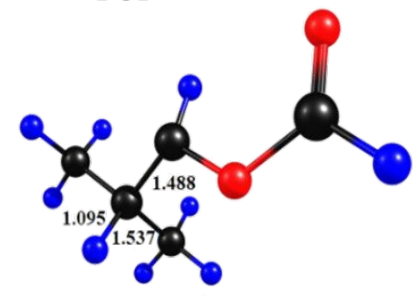

P2
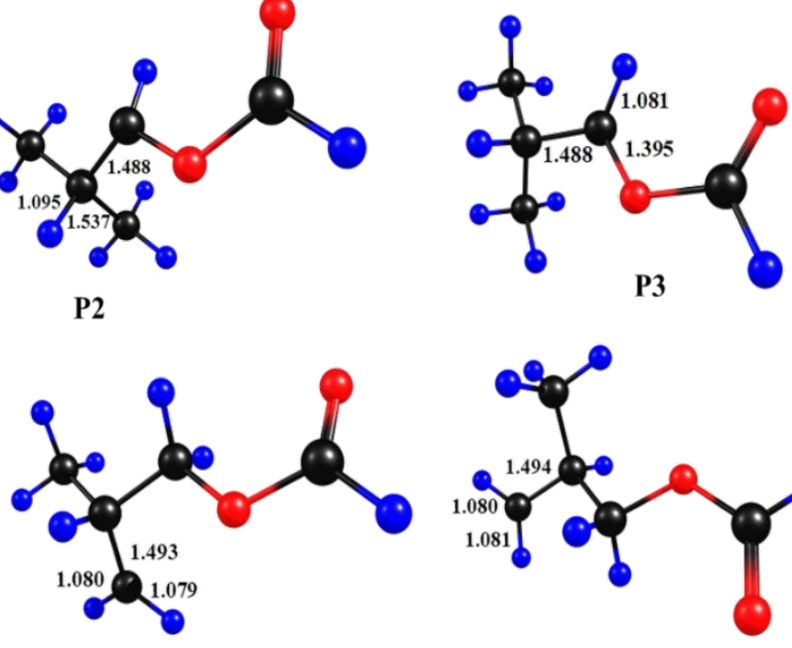

P6

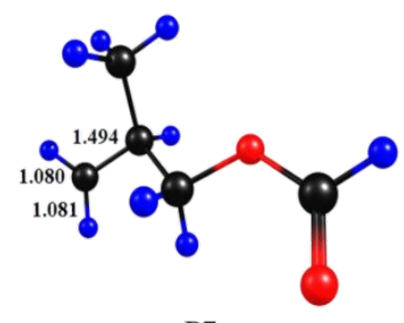

P7 


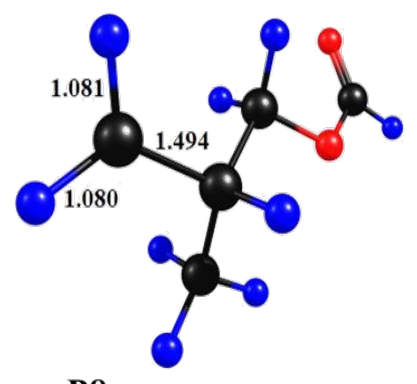

P8

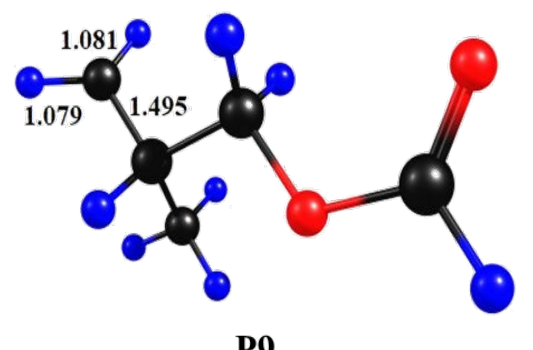

P9

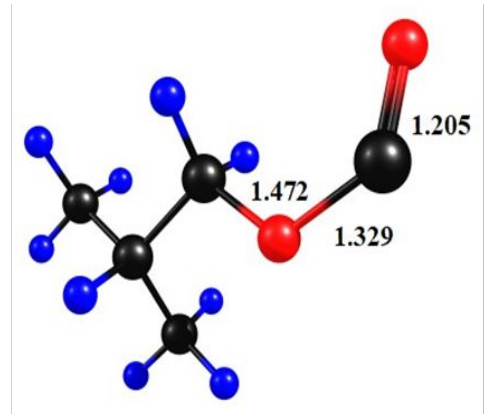

P10

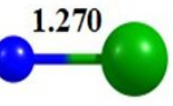

$\mathrm{HCl}$

Figure S4: Optimized geometries of the reactants, pre-reactive complexes, transition states and products for the reaction of $\mathrm{Cl}$ atoms with isobutyl formate obtained at MP2/6-31+G(d,p) level of theory. Black color represents carbon atoms, blue color represents hydrogen atoms, green color represents $\mathrm{Cl}$ atoms and red color represents oxygen atoms. 

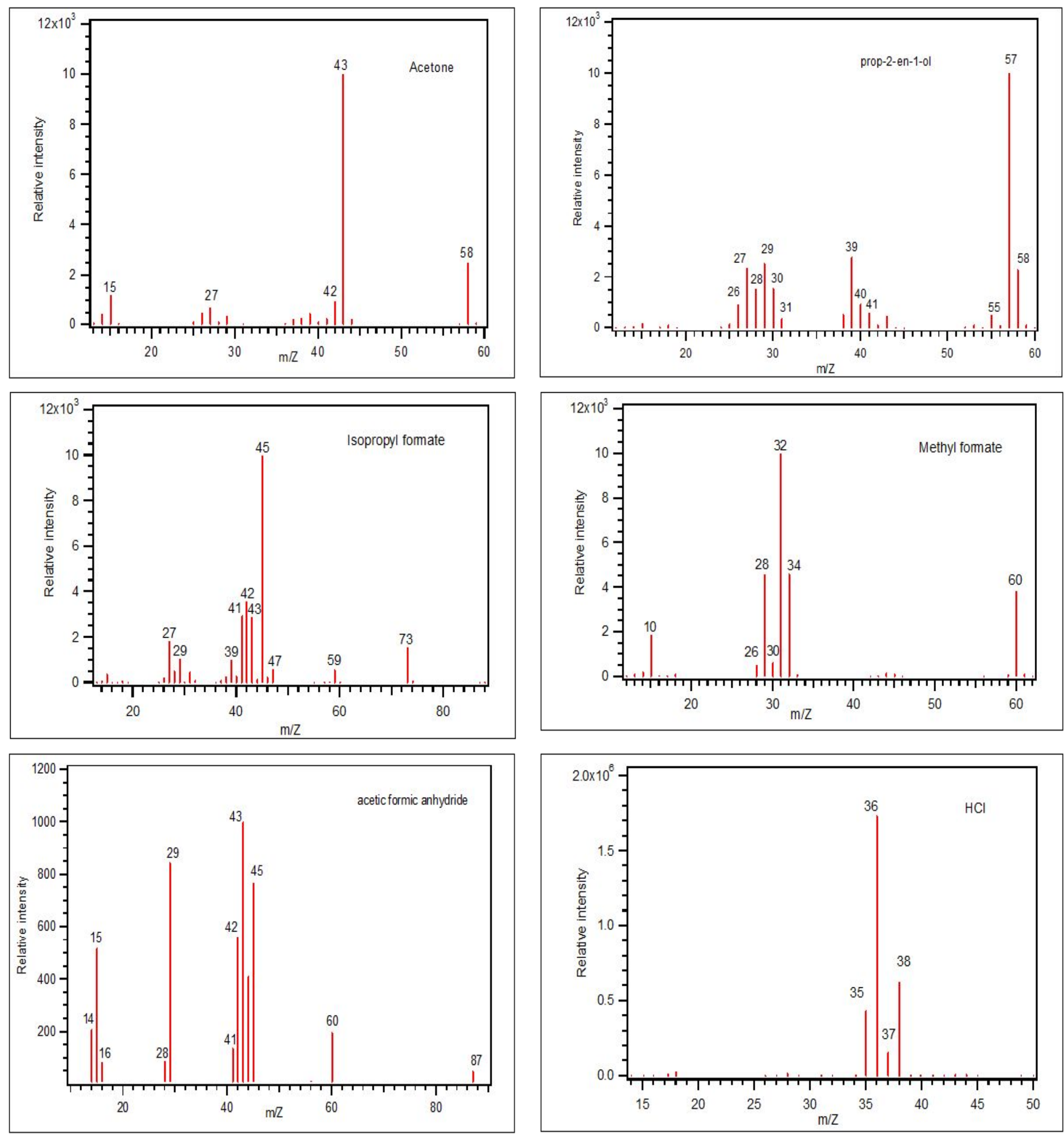

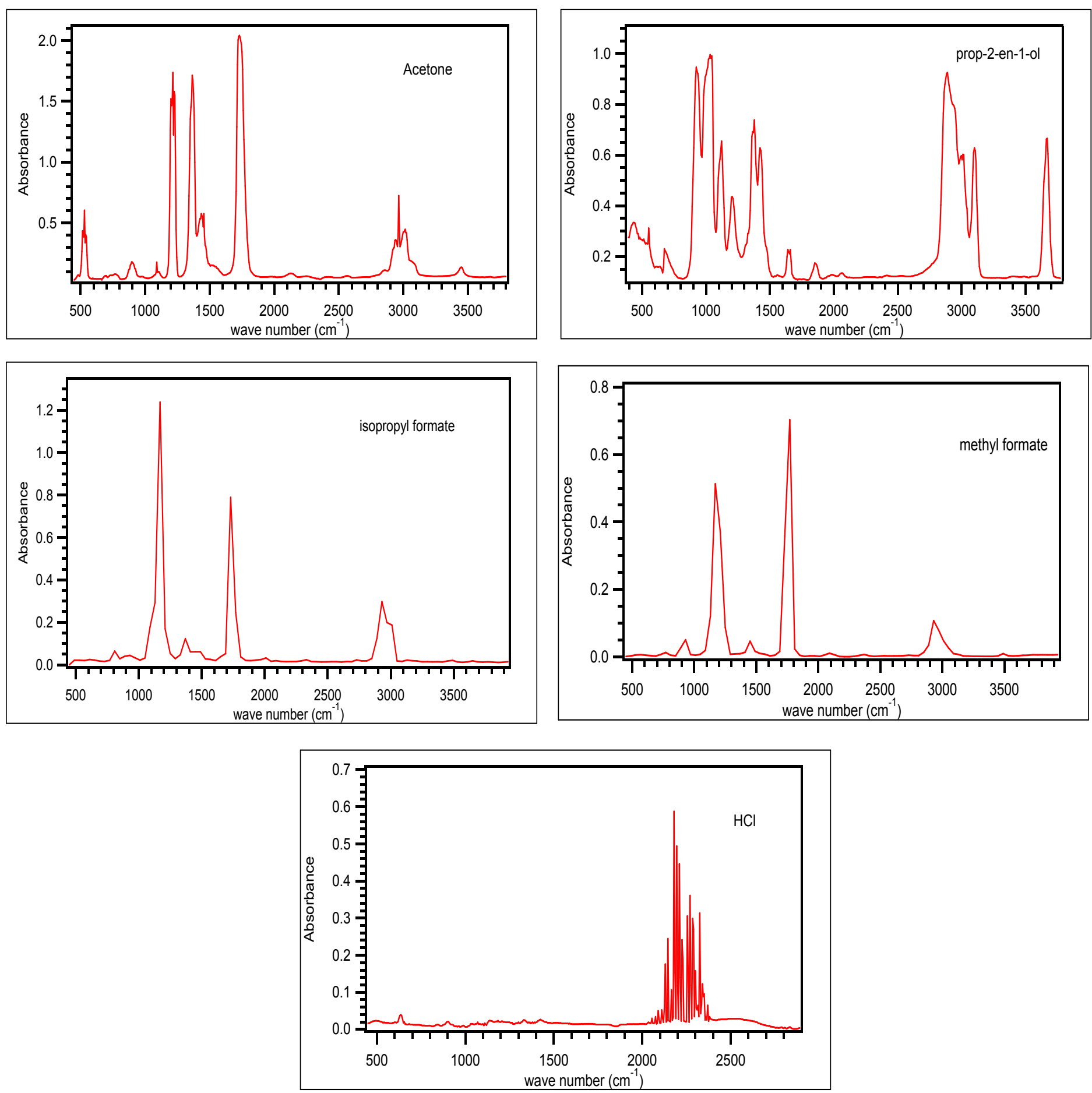
Figure S5: Mass spectrum and IR spectrum of each product obtained for the reaction of isopropyl formate with $\mathrm{Cl}$ atoms.
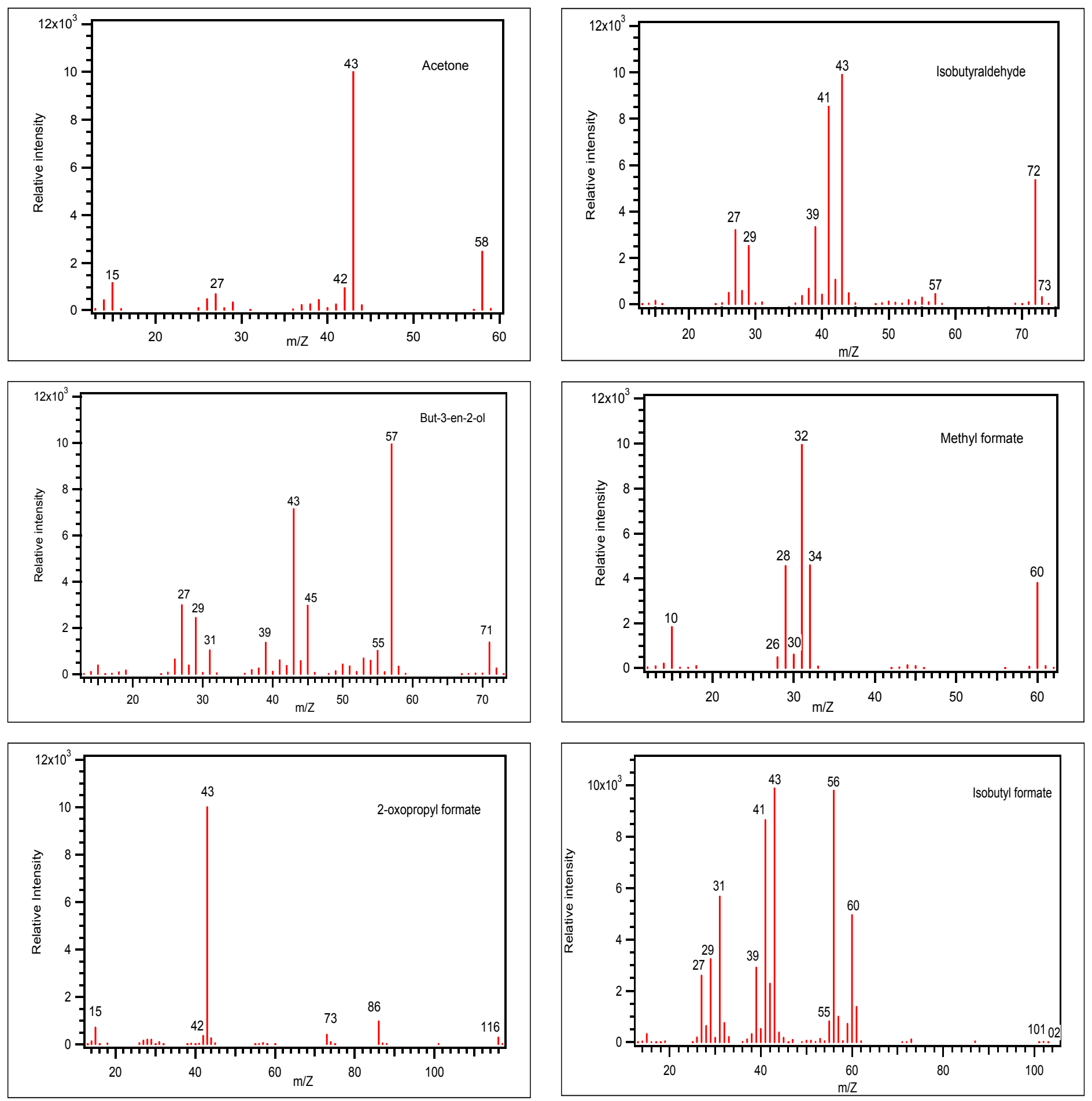

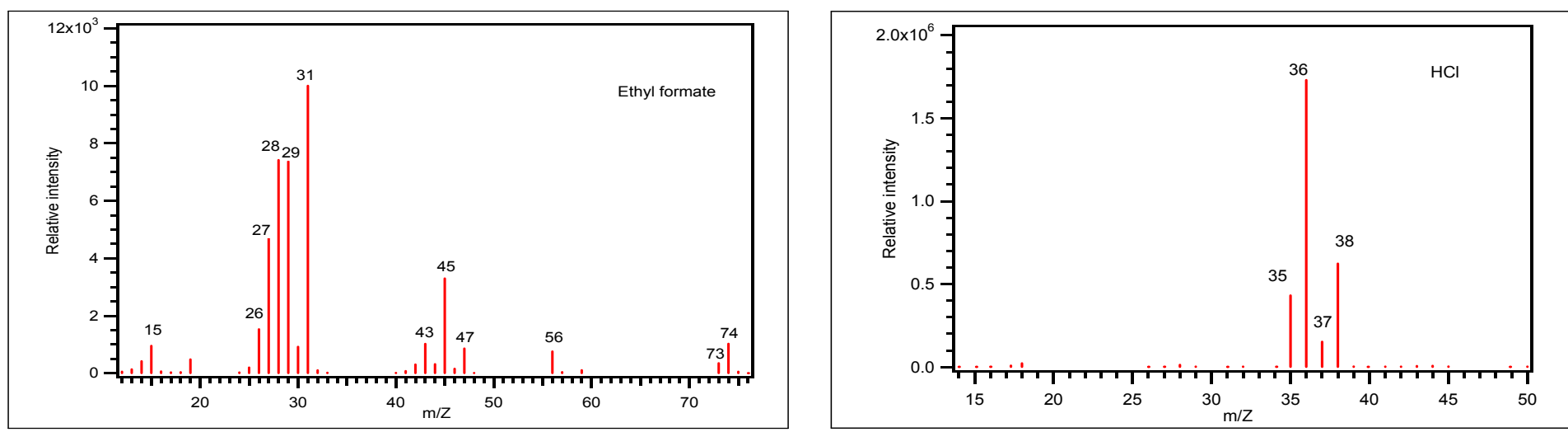

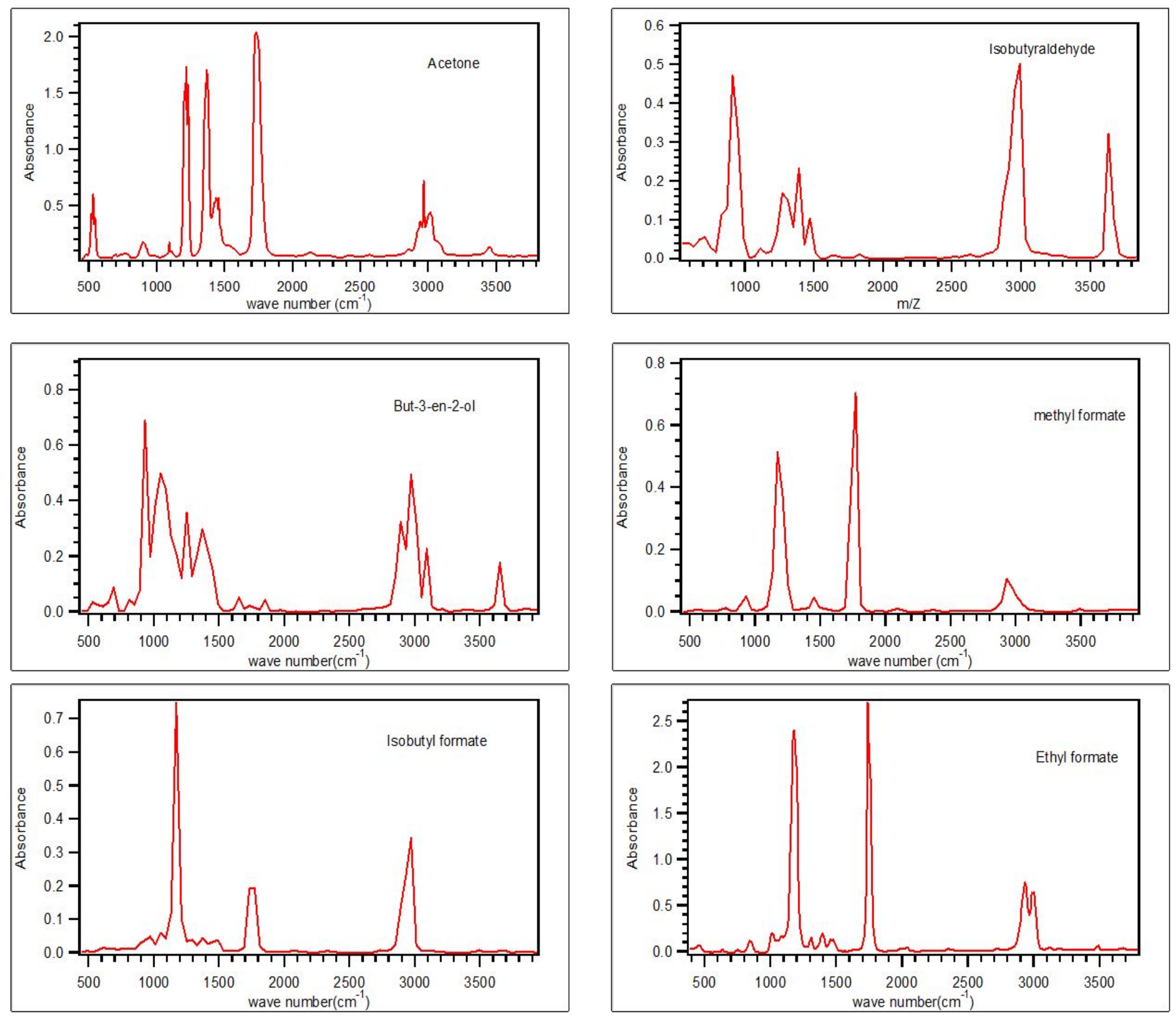


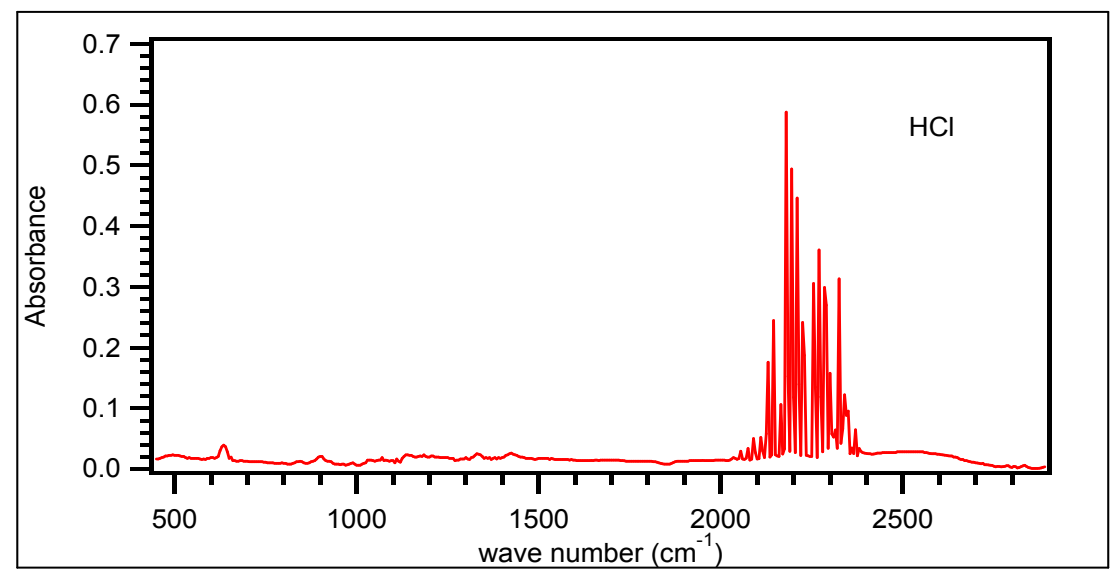

Figure S6: Mass spectrum and IR spectrum of each product obtained for the reaction of isobutyl formate with $\mathrm{Cl}$ atoms. 